\title{
Confronting grand unification with lepton flavour violation, dark matter and LHC data
}

\author{
J. Ellis, ${ }^{a, b, c}$ M.E. Gómez, ${ }^{d}$ S. Lola, ${ }^{e}$ R. Ruiz de Austri $^{f}$ and Q. Shafi ${ }^{g}$ \\ ${ }^{a}$ Theoretical Particle Physics and Cosmology Group, \\ Department of Physics, King's College London, Strand, London WC2R 2LS, U.K. \\ ${ }^{b}$ National Institute of Chemical Physics $\mathscr{G}$ Biophysics, Rävala 10, 10143 Tallinn, Estonia \\ ${ }^{c}$ Theoretical Physics Department, CERN, CH-1211 Geneva 23, Switzerland \\ ${ }^{d}$ Departamento de Ciencias Integradas y Centro de Estudios Avanzados en Física Matemáticas \\ y Computación, Campus El Carmen, Universidad de Huelva, 21071 Huelva, Spain \\ ${ }^{e}$ Department of Physics, University of Patras, 26500 Patras, Greece \\ ${ }^{f}$ Instituto de Física Corpuscular, IFIC-UV/CSIC, Valencia, Spain \\ ${ }^{g}$ Bartol Research Institute, Department of Physics and Astronomy, University of Delaware, \\ Newark, DE 19716, U.S.A. \\ E-mail: John.Ellis@cern.ch, mario.gomez@dfa.uhu.es, \\ magda@physics.upatras.gr, rruiz@ific.uv.es, shafi@bartol.udel.edu
}

ABSTRACT: We explore possible signatures for charged lepton flavour violation (LFV), sparticle discovery at the LHC and dark matter (DM) searches in grand unified theories (GUTs) based on $\mathrm{SU}(5)$, flipped $\mathrm{SU}(5)(\mathrm{FSU}(5))$ and $\mathrm{SU}(4)_{c} \times \mathrm{SU}(2)_{L} \times \mathrm{SU}(2)_{R}$ (4-2-2). We assume that soft supersymmetry-breaking terms preserve the group symmetry at some high input scale, and focus on the non-universal effects on different matter representations generated by gauge interactions at lower scales, as well as the charged LFV induced in Type-1 see-saw models of neutrino masses. We identify the different mechanisms that control the relic DM density in the various GUT models, and contrast their LFV and LHC signatures. The SU(5) and 4-2-2 models offer good detection prospects both at the LHC and in LFV searches, though with different LSP compositions, and the SU(5) and FSU(5) models offer LFV within the current reach. The 4-2-2 model allows chargino and gluino coannihilations with neutralinos, and the former offer good detection prospects for both the LHC and LFV, while gluino coannihilations lead to lower LFV rates. Our results indicate that LFV is a powerful tool that complements LHC and DM searches, providing significant insights into the sparticle spectra and neutrino mass parameters in different models.

KeYWORds: Supersymmetry Phenomenology

ARXIV EPRINT: 2002.11057 


\section{Contents}

1 Introduction 1

2 Non-universal soft supersymmetry breaking in GUT models 2

3 Relic density mechanisms and GUT mass relations 4

4 Lepton-flavour mixing effects and see-saw neutrino masses $\quad 6$

5 LFV, dark matter and the LHC 9

$\begin{array}{lll}5.1 & \mathrm{BR}\left(l_{i} \rightarrow l_{j} \gamma\right) & 10\end{array}$

5.2 Combining $\mu \rightarrow e \gamma$ and LHC bounds $\quad 12$

$\begin{array}{lll}5.3 & \text { LFV signals, SUSY spectroscopy and DM detection } & 15\end{array}$

$\begin{array}{llr}6 & \text { Conclusions } & 19\end{array}$

\section{Introduction}

Experimental and theoretical considerations both require extending the Standard Model (SM) of particle physics, which can neither accommodate massive neutrinos, nor explain the observed baryon asymmetry of the universe, nor provide dark matter [1-4]. Nevertheless, the data from the LHC [5-8] and dark matter searches [9-13] have not yet yielded any positive signature of physics beyond the SM. On the contrary, severe constraints have been derived for the simplest extensions of the SM that address these issues, including the most simplified versions of supersymmetric unified theories. However, supersymmetry (SUSY) continues to have strong theoretical attraction and, among other features, provides a natural candidate for dark matter (DM) $[14,15]$ and facilitates the construction of grand unified theories (GUTs) [16-20]. It is therefore premature to exclude SUSY before studying in more detail non-simplified models that have not yet been explored.

In doing so, flavour physics inevitably plays a crucial role, since it also provides severe bounds on extensions of the SM that would have resulted in exotic manifestations of flavour violation that have not yet been observed. In particular, supersymmetric theories have several possible sources of lepton flavour violation (LFV), which would yield unacceptably large effects unless off-diagonal entries in the sfermion mass matrices were small at some high scale. Even in this case, however, quantum corrections during the running from high scales to low energies would modify this simple structure. This effect is particularly significant in see-saw models for neutrino masses, where the Dirac neutrino Yukawa couplings cannot be diagonalised simultaneously with the charged (s)lepton Yukawa couplings [21]. In this case, the large mixing of neutrino families required by the data also implies that charged LFV may occur at enhanced rates for sufficiently small 
soft supersymmetry-breaking masses. This can occur in rare decays and conversions (e.g., $\mu \rightarrow e \gamma$ and $\tau \rightarrow \mu \gamma, \mu \rightarrow 3 e, \tau \rightarrow e \gamma$ and $\mu-e$ conversion $[22,23]),{ }^{1}$ but also in other processes such as sparticle production at the LHC [26-36] and slepton pair production at a Linear Collider (LC) [37-45], particularly in the decays of the second-lightest neutralino.

In this paper we study the possibilities for LFV, LHC and dark matter searches in models with various grand unification groups. We pay particular attention to comparisons between their respective signatures, and to ways to differentiate between the various schemes in present and future searches. Since the $\mathrm{SO}(10)$ GUT model is now severely constrained by the data, we focus on GUTs based on SU(5), flipped SU(5) (FSU(5)) and $\mathrm{SU}(4)_{c} \times \mathrm{SU}(2)_{L} \times \mathrm{SU}(2)_{R}(4-2-2)$ [46-51]. Similarly to previous works within various GUT models [52-58], we assume that at the unification scale the soft SUSY-breaking terms preserve the group symmetry, and focus on the non-universal effects on different matter representations due to the gauge structures of the groups. We also include the charged LFV induced in these models via Type- 1 see-saw models of neutrino masses with right-handed neutrinos at some high intermediate mass scale.

Different mechanisms that control the relic DM density in the various GUT models, lead to contrasting LFV and LHC signatures. Although the results are sensitive to the scale of the right-handed neutrinos (with larger scales being linked to larger couplings and thus larger flavour violation due to quantum corrections), for similar see-saw parameters, detailed comparisons between different unification schemes can be made. In all cases, coannihilations result to higher LFV rates. We find that the SU(5) and 4-2-2 models have different LSP compositions, but both offer good prospects for detection at both the LHC and in LFV searches. The FSU(5) model also offers LFV within the current reach, for instance in stop and stau coannihilation scenarios. The 4-2-2 model admits novel DM mechanisms such as chargino and gluino coannihilations with neutralinos. The former offers good detection prospects for both the LHC and LFV, whereas gluino coannihilations lead to lower LFV rates, and Higgsino DM models do not predict detectable LFV. In addition, we derive specific correlations between the respective sparticle spectra, providing further input on the experimental signatures that can be expected in each scheme, commenting on the prospects for direct detection of DM as well as LHC and LFV searches.

In section 2 we summarise the basic non-universal features of the GUTs we study that are relevant for our discussion. In section 3 we discuss different mechanisms for determining the relic density of dark matter (DM) in the presence of non-universal SUSYbreaking mass terms. In section 4 we discuss lepton flavour mixing effects in the presence of see-saw neutrinos. In section 5 we look at the branching ratios for rare LFV decays in different DM scenarios, also taking LHC and direct DM searches into account. Finally, our main results and future detection prospects are summarised in section 6 .

\section{Non-universal soft supersymmetry breaking in GUT models}

In our analysis, we assume that SUSY breaking occurs in a hidden sector at some scale $M_{X}>M_{\mathrm{GUT}}$, via a mechanism that generates flavour-blind soft terms in the visible sector.

\footnotetext{
${ }^{1}$ For pioneering studies of $\mu \rightarrow e \gamma$ in supersymmetric models, see [24, 25].
} 
Between the scales $M_{X}$ and $M_{\mathrm{GUT}}$, although the theory still preserves the GUT symmetry, quantum corrections may induce non-universalities for soft terms that belong to different GUT representations, while particles that belong to the same representation have common soft masses.

The soft SUSY-breaking scalar terms for the fields in an irreducible representation $r$ of the unification group are parametrised as multiples of a common scale $m_{0}$ :

$$
m_{r}=x_{r} m_{0},
$$

while the trilinear terms are defined as:

$$
A_{r}=Y_{r} A_{0}, \quad A_{0}=a_{0} m_{0},
$$

where $Y_{r}$ is the Yukawa coupling associated with the representation $r$. We use the standard parametrization with $a_{0}$ a dimensionless factor, which we assume to be representationindependent. Since the two Higgs fields of the MSSM arise from different SU(5) representations, they have in general different soft masses. The situation in the different GUT groups is then as follows:

- $\mathrm{SU}(5)$. In this case, the multiplet assignments are as follows:

$$
\left(Q, u^{c}, e^{c}\right)_{i} \in \mathbf{1 0}_{i},\left(L, d^{c}\right)_{i} \in \overline{\mathbf{5}}_{i}, \nu_{i}^{c} \in \mathbf{1}_{i} .
$$

We assume that the soft terms are the same for all the members of the same representation at the GUT scale, but may be different for the $\mathbf{1 0}$ and $\overline{\mathbf{5}}$ representations. Here we use as reference the common soft SUSY-breaking masses for the fields of the 10, denoted by $m_{10}$. The masses for the other representations are then defined as:

$$
m_{10}=m_{0}, \quad m_{5}=x_{5} \cdot m_{10}, \quad m_{H_{u}}=x_{u} \cdot m_{10}, \quad m_{H_{d}}=x_{d} \cdot m_{10},
$$

and the $A$ terms are specified via a common mass scale:

$$
A_{10,5}=a_{0} m_{0} .
$$

- Flipped SU(5) (FSU(5)). Since the particle assignments are now different, namely:

$$
\left(Q, d^{c}, \nu^{c}\right)_{i} \in \mathbf{1 0}_{i},\left(L, u^{c}\right)_{i} \in \overline{\mathbf{5}}_{i}, e_{i}^{c} \in \mathbf{1}_{i},
$$

and the parametrisation changes to

$$
m_{10}=m_{0}, \quad m_{5}=x_{5} \cdot m_{10} \quad m_{R}=x_{R} \cdot m_{10} \quad m_{H_{u}}=x_{u} \cdot m_{10} \quad m_{H_{d}}=x_{d} \cdot m_{10}
$$

where $x_{R}$ refers to the $\mathrm{SU}(2)$-singlet fields. As previously, the $A$ terms are specified as universal: $A_{0}=a_{0} \cdot m_{0}$.

- 4-2-2 symmetry. In this case, a significant modification arises already in the correlation of gaugino masses, since the embedding of the hypercharge generator in the 4-2-2 group implies:

$$
M_{1}=\frac{3}{5} M_{2}+\frac{2}{5} M_{3},
$$


yielding gluino coannihilations that are absent in models based on other groups [52]. Sfermions are accommodated in 16-dimensional spinor representations, with their common soft mass parameter being $m_{16}$. The electroweak MSSM doublets lie in the 10-dimensional representation with D-term contributions that split their soft masses: $m_{H_{u, d}}^{2}=m_{10}^{2} \pm 2 M_{D}^{2}$. In our notation:

$$
x_{u}=\frac{m_{H_{u}}}{m_{16}}, \quad x_{d}=\frac{m_{H_{d}}}{m_{16}},
$$

with $x_{u}<x_{d}$. In the left-right asymmetric 4-2-2 model, a new parameter is introduced:

$$
x_{L R}=\frac{m_{L}}{m_{R}},
$$

where $m_{L}$ is the mass of the left-handed sfermions (that preserve the definition of $\left.m_{16}=m_{0}\right)$, and $m_{R}$ is the mass of the corresponding right-handed ones.

\section{Relic density mechanisms and GUT mass relations}

We assume the following relic density constraint [4]:

$$
\Omega_{\chi} h^{2}=0.1186 \pm 0.0031,
$$

with a (fixed) theoretical uncertainty of $\tau=0.012$, (following ref. [59]) to account for numerical uncertainties in the relic density calculation. This narrow range on the relic density imposes a strong constraint on the DM candidate and the mechanisms that determine its density.

It is well known that particular mass relations must be present in the supersymmetric spectrum if the required amount of relic dark matter is provided by neutralinos. In addition to mass relations, we use the neutralino composition to classify the relevant points of the supersymmetric parameter space. The higgsino fraction of the lightest neutralino mass eigenstate is characterized by the quantity

$$
h_{f} \equiv\left|N_{13}\right|^{2}+\left|N_{14}\right|^{2},
$$

where the $N_{i j}$ are the elements of the unitary mixing matrix that correspond to the higgsino mass states. Thus, we classify the points that pass the relic density constraint discussed above according to the following criteria:

\section{Higgsino DM.}

$$
h_{f}>0.1,\left|m_{A}-2 m_{\chi}\right|>0.1 m_{\chi} .
$$

The first condition in (3.3) ensures that the lightest neutralino is higgsino-like and, as we discuss later, the lightest chargino $\chi_{1}^{ \pm}$is almost degenerate in mass with $\chi_{1}^{0}$. The couplings to the SM gauge bosons are not suppressed and $\chi_{1}^{0}$ pairs have large cross sections for annihilation into $W^{+} W^{-}$and $Z Z$ pairs, which may reproduce the observed value of the relic DM abundance. Clearly, coannihilation channels involving $\chi_{1}^{ \pm}$and $\chi_{2}^{0}$ also contribute. The second condition in (3.3) implies that the DM density is not controlled by rapid annihilation through $s$-channel resonances. 


\section{$\boldsymbol{A} / \boldsymbol{H}$ resonances.}

$$
\left|m_{A}-2 m_{\chi}\right| \leq 0.1 m_{\chi} .
$$

This condition ensures that the correct value of the relic DM abundance is achieved thanks to $s$-channel annihilation, enhanced by the resonant heavy neutral Higgs ( $A$ and $H$ ) propagators. The thermal average $\left\langle\sigma_{a n n} v\right\rangle$ spreads out the peak in the cross section, so that neutralino masses for which $2 m_{\chi} \simeq m_{A}$ is not exactly realized can also experience resonant annihilations.

$\tilde{\tau}-\chi_{1}^{0}$ coannihilations.

$$
h_{f}<0.1, \quad\left(m_{\tilde{\tau}_{1}}-m_{\chi}\right) \leq 0.1 m_{\chi}
$$

The first condition in (3.5) ensures that the neutralino is bino-like, in which case annihilation into leptons through $t$-channel slepton exchange is suppressed, and when the second condition in (3.5) is satisfied coannihilations involving the nearly-degenerate $\tilde{\tau}_{1}$ enhance the thermal-averaged effective cross section.

$\tilde{\tau}-\tilde{\nu}_{\tau}-\chi_{1}^{0}$ coannihilations.

$$
h_{f}<0.1, \quad\left(m_{\tilde{\tau}_{1}}-m_{\chi}\right) \leq 0.1 m_{\chi}, \quad\left(m_{\tilde{\nu}_{\tau}}-m_{\chi}\right) \leq 0.1 m_{\chi} .
$$

This is similar to the previous case, but also the $\nu_{\tilde{\tau}}$ is nearly degenerate in mass with the $\tilde{\tau}_{1}$.

$\tilde{t_{1}}-\chi_{1}^{0}$ coannihilations.

$$
h_{f}<0.15, \quad\left(m_{\tilde{t}_{1}}-m_{\chi}\right) \leq 0.1 m_{\chi} .
$$

In this case the $\tilde{t}_{1}$ is light and nearly degenerate with the bino-like neutralino. These coannihilations are present in the flipped SU(5) model and in the 4-2-2 model, but not $\mathrm{SU}(5)$.

In previous work, we had found that the 4-2-2 model may be distinguished clearly from the other GUT groups, due to the appearance of three additional modes of coannihilation that are not present in other groups:

$\tilde{\chi}^{+}-\chi_{1}^{0}$ coannihilations.

$$
h_{f}<0.1, \quad\left(m_{\tilde{\chi}^{+}}-m_{\chi}\right) \leq 0.1 m_{\chi} .
$$

In this case the Higgsino component in the LSP is small, but the lightest chargino is light and nearly degenerate with the bino-like neutralino.

$\tilde{\boldsymbol{g}}-\chi_{1}^{0}$ coannihilations.

$$
h_{f}<0.1, \quad\left(m_{\tilde{g}}-m_{\chi}\right) \leq 0.1 m_{\chi} .
$$

In this case the gluino can be relatively light and nearly degenerate with the bino-like neutralino. 
$\tilde{b}-\chi_{1}^{0}$ coannihilations.

$$
h_{f}<0.1, \quad\left(m_{\tilde{b}}-m_{\chi}\right) \leq 0.1 m_{\chi}
$$

in this case, in the presence of the LR asymmetry, the $\tilde{b}$ can be light and nearly degenerate with a bino-like neutralino [60].

\section{Lepton-flavour mixing effects and see-saw neutrino masses}

In what follows, we supplement the previous framework with a see-saw mechanism so as to incorporate neutrino masses [61, 62]. We consider a high-scale see-saw mechanism in which, in order to obtain order $0.1 \mathrm{eV}$ masses for the neutrinos, this scale should be around $10^{14} \mathrm{GeV}$ (assuming electroweak-scale Dirac neutrino masses). Such a mechanism can be realized by extending the MSSM with renormalizable interactions in three scenarios: type I [63-69] that requires singlet $\mathrm{RH}$ neutrinos, type II $[70,71]$ that requires scalar $\mathrm{SU}(2)_{L}$ triplets and type III [72] that requires fermionic $\mathrm{SU}(2)_{L}$ triplets. Here we focus on the type-I see-saw, in which the additional singlet $\mathrm{RH}$ neutrino fields do not affect the running of the gauge couplings and therefore fit well in our unification schemes. Some examples of the phenomenology of type II and type III models can be found in refs. [73, 74].

We use the following superpotential:

$$
W=W_{\mathrm{MSSM}}+Y_{\nu}^{i j} \epsilon_{\alpha \beta} H_{2}^{\alpha} N_{i}^{c} L_{j}^{\beta}+\frac{1}{2} M_{N}^{i j} N_{i}^{c} N_{j}^{c},
$$

where $W_{\text {MSSM }}$ is the MSSM superpotential and the $N_{i}^{c}$ are additional superfields that contain the three singlet (right-handed) neutrinos, $\nu_{R i}$, and their scalar partners, $\tilde{\nu}_{R i}$, and $M_{N}^{i j}$ denotes the $3 \times 3$ Majorana mass matrix for the heavy right-handed neutrinos. The full set of soft SUSY-breaking terms is given by

$$
-L_{\mathrm{soft}, \mathrm{SI}}=-L_{\mathrm{soft}}+\left(m_{\tilde{\nu}}^{2}\right)_{j}^{i} \tilde{\nu}_{R i}^{*} \tilde{\nu}_{R}^{j}+\left(\frac{1}{2} B_{\nu}^{i j} M_{N}^{i j} \tilde{\nu}_{R i}^{*} \tilde{\nu}_{R j}^{*}+A_{\nu}^{i j} h_{2} \tilde{\nu}_{R i}^{*} \tilde{l}_{L j}+\text { h.c. }\right)
$$

where $L_{\text {soft }}$ contains the MSSM soft SUSY-breaking masses, and $\left(m_{\tilde{\nu}}^{2}\right)_{j}^{i}, A_{\nu}^{i j}$ and $B_{\nu}^{i j}$ are the new soft SUSY-breaking parameters in the see-saw sector.

The see-saw mechanism yields three heavy neutral mass eigenstates that are mainly right-handed and decouple at a high energy scale, with masses that we denote as $M_{N}$. Below this scale, the effective theory contains the MSSM plus a higher-dimensional operator that provides masses for the light neutrinos, which are mainly left-handed:

$$
W=W_{\mathrm{MSSM}}+\frac{1}{2}\left(Y_{\nu} L H_{2}\right)^{T} M_{N}^{-1}\left(Y_{\nu} L H_{2}\right)
$$

As the right-handed neutrinos decouple at their respective mass scales, at low energy we have the same particle content and mass matrices as in the MSSM. This framework naturally accommodates neutrino oscillations that are consistent with experimental data [75-83]. At the electroweak scale an effective Majorana mass matrix for light neutrinos,

$$
m_{\mathrm{eff}}=-\frac{1}{2} v_{u}^{2} Y_{\nu} \cdot M_{N}^{-1} \cdot Y_{\nu}^{T}
$$


arises from the Dirac neutrino Yukawa coupling matrix $Y_{\nu}$ (with entries that can be assumed to be of the same order of magnitude as the charged-lepton and quark Yukawa couplings), and the heavy Majorana masses $M_{N}$.

We observe from (4.1) that we can rotate the fields $L_{i}$ and $N_{i}^{c}$ in such a way that the matrices of the lepton Yukawa couplings, $Y_{l}^{i j}$, and of the right-handed neutrinos, $M_{N}^{i j}$, become diagonal. However, in this basis, the neutrino Yukawa couplings $Y_{\nu}^{i j}$ are not in general diagonal, giving rise to lepton-flavour-violating (LFV) effects [84-90]. It is important to note here that lepton-flavour conservation is not a consequence of the SM gauge symmetry, even in the absence of the right-handed neutrinos. Consequently, slepton mass terms can violate lepton-flavour conservation in a manner consistent with the gauge symmetry. Indeed, the scale of LFV can be identified with the EW scale, much lower than the right-handed neutrino scale, $M_{R}$, which we assume to be common, for simplicity. In the basis where the charged-lepton Yukawa matrix $Y_{\ell}$ is diagonal, the soft slepton mass matrix acquires corrections that contain off-diagonal contributions from the RGE running from $M_{\mathrm{GUT}}$ down to $M_{R}$, which are of the following form in the leading-log approximation [91]:

$$
\begin{aligned}
\left(m_{\tilde{L}}^{2}\right)_{i j} & \sim \frac{1}{16 \pi^{2}}\left(6 m_{0}^{2}+2 A_{0}^{2}\right)\left(Y_{\nu}^{\dagger} Y_{\nu}\right)_{i j} \log \left(\frac{M_{\mathrm{GUT}}}{M_{R}}\right), \\
\left(m_{\tilde{e}}^{2}\right)_{i j} & \sim 0, \\
\left(A_{l}\right)_{i j} & \sim \frac{3}{8 \pi^{2}} A_{0} Y_{l i}\left(Y_{\nu}^{\dagger} Y_{\nu}\right)_{i j} \log \left(\frac{M_{\mathrm{GUT}}}{M_{R}}\right) .
\end{aligned}
$$

Below $M_{R}$, the off-diagonal contributions remain almost unchanged. Their magnitude depends on the structure of $Y_{\nu}$ at $M_{R}$, in a basis where $Y_{l}$ and $M_{N}$ are diagonal. Using the approach of $[89,92]$ a generic form for $Y_{\nu}$ that contains all neutrino experimental information can be obtained:

$$
Y_{\nu}=\frac{\sqrt{2}}{v_{u}} \sqrt{M_{N}^{\delta}} O_{R} \sqrt{m_{\nu}^{\delta}} U^{\dagger}
$$

where $O_{R}$ is a general orthogonal matrix and $M_{N}^{\delta}$ and $m_{\nu}^{\delta}$ denote the diagonalized heavy and light Majorana neutrino mass matrices, respectively. In this basis the matrix $U$ can be identified with the Pontecorvo-Maki-Nakagawa-Sakata (PMNS) matrix, $U_{\text {PMNS: }}$ :

$$
m_{\nu}^{\delta}=U^{T} m_{\mathrm{eff}} U
$$

Assuming that the observed neutrino oscillations can be attributed to hierarchical neutrino masses, we have $m_{\nu}^{\delta}=\operatorname{Diag}\left(1.1 \cdot 10^{-3}, 8 \cdot 10^{-3}, 5 \cdot 10^{-2}\right) \mathrm{eV}$. For $Y_{\nu}$ couplings of order one, the RH neutrinos can take values as large as $10^{14} \mathrm{GeV}$. The LFV BR's decrease with the RH neutrino scale. However, the matrix $O_{R}$ is associated with the flavor structure of the $\mathrm{RH}$ neutrino mass matrix, which must be nontrivial so as to provide a scenario for baryogenesis through leptogenesis, which typically requires masses of order $10^{8}-10^{9} \mathrm{GeV}[93,94]$. It may also induce cancellations in the LFV BRs that may allow $\mathrm{RH}$ neutrino masses above the $10^{14} \mathrm{GeV}$ scale while respecting the current constraints. To illustrate this point, we consider real see-saw parameters and parametrize the matrix $O_{R}$ 
with three real angles $\theta_{1}, \theta_{2}, \theta_{3}$, following the notation of ref. [89]:

$$
O_{R}=R_{12}\left(\hat{\theta}_{3}\right) \cdot R_{13}\left(\hat{\theta}_{2}\right) \cdot R_{23}\left(\hat{\theta}_{1}\right)
$$

where

$$
R_{23}=\left(\begin{array}{ccc}
1 & 0 & 0 \\
0 & \hat{c}_{1} & \hat{s}_{1} \\
0 & \hat{s}_{1} & \hat{c}_{1}
\end{array}\right) ; R_{13}=\left(\begin{array}{ccc}
\hat{c}_{2} & 0 & \hat{s}_{2} \\
0 & 1 & 0 \\
\hat{s}_{2} & 0 & \hat{c}_{2}
\end{array}\right) ; R_{12}=\left(\begin{array}{ccc}
\hat{c}_{3} & \hat{s}_{3} & 0 \\
\hat{s}_{3} & \hat{c}_{3} & 0 \\
0 & 0 & 1
\end{array}\right)
$$

We denote $\sin \left(\hat{\theta}_{i}\right)$ and $\cos \left(\hat{\theta}_{i}\right)$ as $\hat{s}_{i}$ and $\hat{c}_{i}$ respectively $(i=1,2,3)$.

For the matrix $U$, we consider as an illustrative example the Harrison, Perkins, and Scott (HPS) mixing matrix [95]:

$$
U=\left(\begin{array}{ccc}
\sqrt{\frac{2}{3}} & \frac{1}{\sqrt{3}} & 0 \\
\frac{-1}{\sqrt{6}} & \frac{1}{\sqrt{3}} & \frac{1}{\sqrt{2}} \\
\frac{-1}{\sqrt{6}} & \frac{1}{\sqrt{3}} & \frac{-1}{\sqrt{2}}
\end{array}\right)
$$

In order to determine the slepton mixing parameters, we need a specific form of the product $Y_{\nu}^{\dagger} Y_{\nu}$, shown in (4.5). Even with the assumption of hierarchical light neutrinos and a fixed $U$ matrix we can still have different predictions for $\operatorname{BR}\left(l_{j} \rightarrow l_{i}+\gamma\right)$ depending on the model used for RH neutrino masses. For instance, in the case of SU(5) models several examples are provided in ref. [84]. The case of degenerate $\mathrm{RH}$ neutrinos implies hierarchical $Y_{\nu}$ matrices, since they inherit the neutrino mass hierarchy, while the BRs are independent of the matrix $O_{R}$. In the case of hierarchical RH neutrinos, the matrix $Y_{\nu}$ can have large entries even for the first two generations, increasing the predicted BRs. However, the matrix $O_{R}$ may induce large cancellations that can result in lower BRs with larger $\mathrm{RH}$ neutrino masses and couplings in the case of degenerate $\mathrm{RH}$ neutrino masses. This behavior can be understood by comparing the predictions for $\operatorname{BR}(\mu \rightarrow e \gamma)$ in the case of degenerate and hierarchical RH neutrinos.

In the case of degenerate right-handed neutrinos $\left(M_{i}=M_{N}\right)$ :

$$
Y_{\nu}=Y_{0} \sqrt{\frac{m_{\nu}^{\delta}}{m_{\nu_{3}}}} U^{\dagger}
$$

where $Y_{0}=\sqrt{2} / v_{u} \sqrt{M_{N} \cdot m_{\nu_{3}}}$. For the hierarchical case, assuming that $m_{\nu_{1}} \sim 0$ and that $M_{1}, M_{2} \ll M_{3}$, using $U$ values from equation (4.10) and the generic rotation (4.9), we find that $Y_{\nu}$ only depends on $\hat{\theta}_{1}$ :

$$
Y_{\nu}=\bar{Y}_{0}\left(\begin{array}{ccc}
0 & 0 & 0 \\
0 & 0 & 0 \\
\sqrt{\frac{m_{\nu_{2}}}{m_{\nu_{3}}}} \cdot \frac{\hat{s}_{1}}{\sqrt{3}} \frac{\hat{c}_{1}}{\sqrt{2}}+\sqrt{\frac{m_{\nu_{2}}}{m_{\nu_{3}}}} \cdot \frac{\hat{s}_{1}}{\sqrt{3}}-\frac{\hat{c}_{1}}{\sqrt{2}}+\sqrt{\frac{m_{\nu_{2}}}{m_{\nu_{3}}}} \cdot \frac{\hat{s}_{1}}{\sqrt{3}}
\end{array}\right)
$$

where $Y_{0}=\sqrt{2} / v_{u} \sqrt{M_{3} \cdot m_{\nu_{3}}} \cdot \hat{c}_{2}$. 


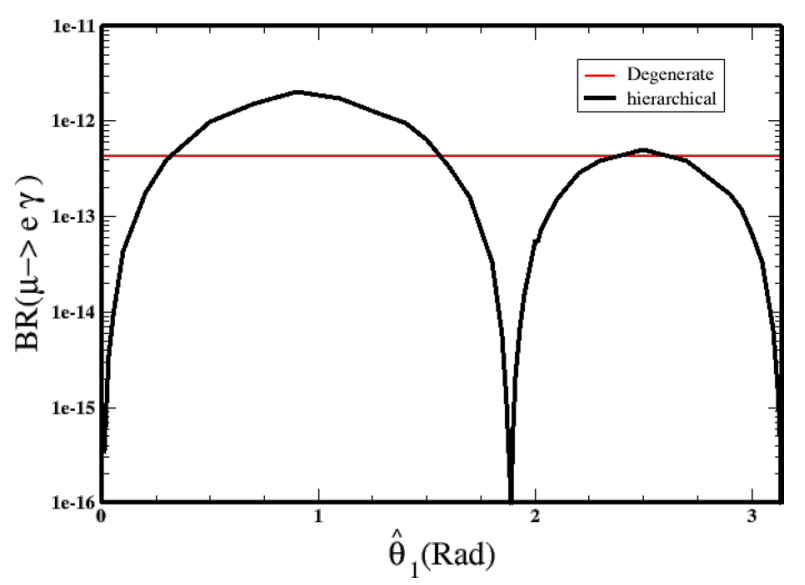

Figure 1. $\operatorname{BR}(\mu \rightarrow e \gamma)$ vs $\hat{\theta}_{1}$ under two different assumptions for the right-handed neutrinos using the CMSSM with $m_{0}=650 \mathrm{GeV}, m_{1 / 2}=700 \mathrm{GeV}, A_{0}=-1400 \mathrm{GeV}$ and $\tan \beta=40, \mu>0$ and $M_{3}=2.5 \cdot 10^{12} \mathrm{GeV}$.

Figure 1 shows the prediction for $\operatorname{BR}(\mu \rightarrow e \gamma)$ under both assumptions for the right-handed neutrinos, assuming the CMSSM with $m_{0}=650 \mathrm{GeV}, m_{1 / 2}=700 \mathrm{GeV}$, $A_{0}=-1400 \mathrm{GeV}$ and $\tan \beta=40, \mu>0$ and $M_{3}=2.5 \cdot 10^{12} \mathrm{GeV}$. For the case of hierarchical right-handed neutrinos we assume $\cos \left(\hat{\theta}_{2}\right)=1$. It is easy to conclude that assuming hierarchical light neutrinos and a common scale for the right-handed neutrinos provides a simple benchmark. In this case, using (4.6), we find

$$
Y_{\nu}^{\dagger} Y_{\nu}=\frac{2}{v_{u}^{2}} M_{R} U m_{\nu}^{\delta} U^{\dagger}
$$

Under the assumption of common masses for the heavy Majorana neutrinos, the LFV effects are independent of the matrix $O_{R}$. On the other hand, the predicted curve of the hierachical case in figure 1 depends on $\hat{\theta}_{1}$ as $\left(Y_{\nu}^{\dagger} Y_{\nu}\right)_{12}^{2}$, where $Y_{\nu}$ is given in eq. (4.12).

\section{LFV, dark matter and the LHC}

We perform parameter space scans similar to those in $[52,57,58]$, where the initial conditions of the soft terms are determined by a unification group that breaks at $M_{\mathrm{GUT}}$ (defined as the scale where the $g_{1}$ and $g_{2}$ couplings meet, while $g_{3}\left(M_{\mathrm{GUT}}\right)$ is obtained by requiring $\left.\alpha_{s}\left(M_{Z}\right)=0.1187\right)$. For our analysis we use the Superbayes [96-98], package to perform statistical inference of SUSY models, which is linked to SoftSusy [99, 100] to compute the SUSY spectrum, to MicrOMEGAs $[101,102]$ and DarkSUSY $[103,104]$ to compute DM observables, to SuperIso $[105,106]$ to compute flavour physics and the muon anomalous magnetic moment $g-2$. The MultiNest [107-109] algorithm is used to scan the parameter space and identify regions compatible with the data.

We have scanned the parameter spaces of the three GUT groups over the broad ranges of parameters shown in table 1, including soft SUSY-breaking terms up to $10 \mathrm{TeV}$, with the results that we now discuss. In addition to the dark matter density constraint mentioned 


\begin{tabular}{|c|ccc|}
\hline SUSY parameters & $4-2-2$ & SU(5) & FSU(5) \\
\hline $100 \mathrm{GeV} \leq m_{0} \leq 10 \mathrm{TeV}$ & $0 \leq x_{u} \leq 2$ & $0 \leq x_{u} \leq 2$ & $0 \leq x_{u} \leq 2$ \\
$50 \mathrm{GeV} \leq m_{1 / 2}\left(M_{2}\right.$ in $\left.4-2-2\right) \leq 10 \mathrm{TeV}$ & $0 \leq x_{d} \leq 2$ & $0 \leq x_{d} \leq 2$ & $0 \leq x_{d} \leq 2$ \\
$-10 \mathrm{TeV} \leq A_{0} \leq 10 \mathrm{TeV}$ & $0 \leq x_{L R} \leq 2$ & $0 \leq x_{5} \leq 2$ & $0 \leq x_{5} \leq 2$ \\
$2 \leq \tan \beta \leq 65$ & & & $0 \leq x_{R} \leq 2$ \\
$-3000 \mathrm{GeV} \leq M_{3}$ (in $\left.4-2-2\right) \leq 10 \mathrm{TeV}$ & & & \\
\hline
\end{tabular}

Table 1. Parameter ranges sampled in our scan of the parameter spaces of the GUT models we study.

above (3.1), we impose the following constraints:

$$
123 \mathrm{GeV} \leq m_{h} \leq 127 \mathrm{GeV},
$$

which includes an allowance for the theoretical uncertainty in the calculation of $m_{h}$ in the CMSSM, which is computed using [99, 100]. We extract the following B-physics constraints from [110]:

$$
1.1 \times 10^{-9} \leq \mathrm{BR}\left(B_{s} \rightarrow \mu^{+} \mu^{-}\right) \leq 6.2 \times 10^{-9},
$$

which accommodates the range allowed experimentally at the $2-\sigma$ level,

$$
2.99 \times 10^{-4} \leq \mathrm{BR}\left(B \rightarrow X_{s} \gamma\right) \leq 3.87 \times 10^{-4},
$$

which also covers the $2-\sigma$ experimental range, and

$$
0.15 \leq \frac{\operatorname{BR}\left(B_{u} \rightarrow \tau \nu_{\tau}\right)_{\mathrm{MSSM}}}{\operatorname{BR}\left(B_{u} \rightarrow \tau \nu_{\tau}\right)_{\mathrm{SM}}} \leq 2.41
$$

which covers the 3- $\sigma$ experimental range. These constraints are implemented as described in [98].

In addition, we impose the constraints on the spin-independent (SI) neutralino-nucleon cross-section provided by the LUX [9], Xenon-1T [11] and PandaX experiments [12].

\section{$5.1 \quad \operatorname{BR}\left(l_{i} \rightarrow l_{j} \gamma\right)$}

The processes $l_{i} \rightarrow l_{j} \gamma$ with $i \neq j$ are allowed at potentially observable levels in SUSY models with flavour mixing among leptons and their scalar partners. In the CMSSM this mixing does not occur, due to the assumption of universal soft terms at the GUT scale. However, this simple SUSY extension of the SM cannot explain neutrino flavour oscillations and, when the model is supplemented with a mechanism to account for them, flavour oscillations of charged leptons also occur. In the MSSM supplemented by a type-I see-saw as described in the previous section, which is compatible with the available neutrino data, the uncertainties in the latter may lead to LFV predictions that can differ by several orders of magnitude. Our target in this work, therefore, is not only to analyze the possibility of observing LFV in current experiments, but also to understand the impact of the bounds on $\mathrm{BR}(\mu \rightarrow e \gamma)$ on the perspectives for LHC data. In the simplified see-saw scenario presented in section 4 , we must still specify the following parameters: 

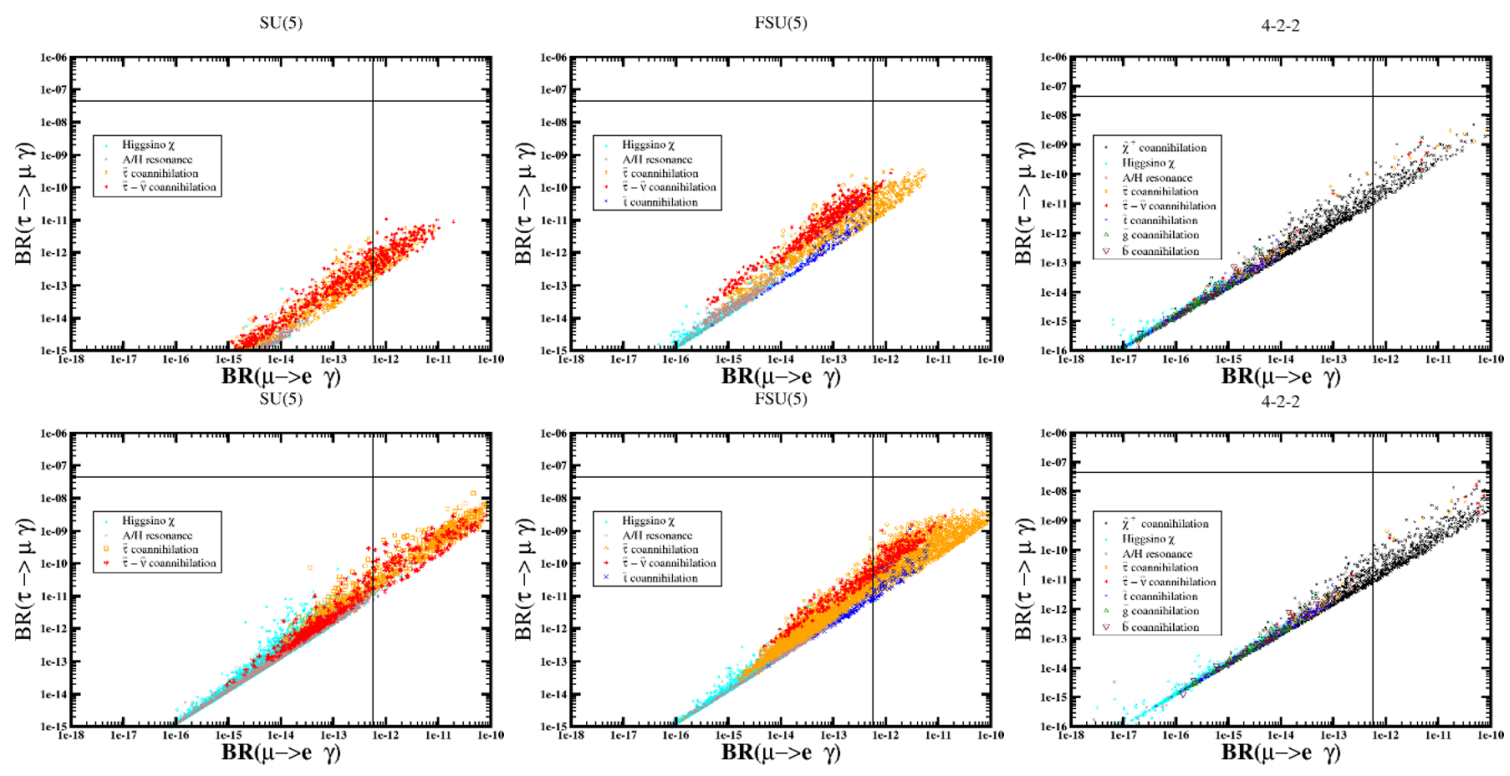

Figure 2. Predictions for $\mathrm{BR}(\tau \rightarrow \mu \gamma)$ and $\mathrm{BR}(\mu \rightarrow e \gamma)$ for (from left to right) the $\mathrm{SU}(5), \operatorname{FSU}(5)$ and 4-2-2 models. The upper (lower) panels assume $M_{N}=2.5 \cdot 10^{12}\left(M_{N}=10^{13} \mathrm{GeV}\right)$. The symbols correspond to classes of models representing the DM scenarios described in the text, which are indicated in the plot legends.

- The right-handed neutrino scale, which we assume to be common for all generations.

- Lepton-slepton mixings parametrized by a matrix similar to the PMNS matrix at the GUT scale. We fix the entries assuming that they are real and that their values are such that the neutrino observables are predicted at their experimental central values. In this simple scheme, the product $Y_{\nu}^{\dagger} Y_{\nu}$ is defined by the PNMS matrix as in (4.13).

- SUSY soft masses are flavour-independent at the GUT scale. However, we allow the sfermions belonging to different representations of the unification group to have different soft masses.

The first two points are discussed in this section, as illustrated in figure 2, whereas the third point requires a more elaborate treatment, and we dedicate the two subsequent sections to it.

For fixed light neutrino masses, eq. (4.4) links the ratio of the square of the Yukawa couplings to the right-handed neutrino masses. We see that higher right-handed neutrino masses imply, in general, higher Yukawa couplings and larger mixings of the scalar sleptons in eq. (4.5), and hence larger LFV branching ratios (BRs). Figure 2 compares model predictions with the experimental upper limits on $\operatorname{BR}(\tau \rightarrow \mu \gamma)$ and $\operatorname{BR}(\mu \rightarrow e \gamma)$. Comparing the upper and lower panels, we can understand how an increase in $M_{R}$ by a factor of 4 would imply the exclusion of many models by the current bound on $\operatorname{BR}(\mu \rightarrow e \gamma)$. For the rest of our analysis, we use $M_{R}=2.5 \cdot 10^{12} \mathrm{GeV}$. In this case, most of the points that can be explored at the LHC will predict $\operatorname{BR}(\mu \rightarrow e \gamma)$ between the current upper limit and a possible future sensitivity one order of magnitude lower. 
The correlation between $\operatorname{BR}(\tau \rightarrow \mu \gamma)$ and $\operatorname{BR}(\mu \rightarrow e \gamma)$ is almost linear, with the prediction of the first being larger than the second by a factor of 10, while the experimental bounds are five orders of magnitude apart. In our study we fixed $Y_{\nu}^{\dagger} Y_{\nu}$ from the PMNS matrix, requiring common right-handed neutrino masses. Although this cannot be considered general, the values of LFV tau decays are maximized by large 2-3 mixing in the PNMS matrix. Furthermore, in SU(5) group symmetries can relate the PMNS and Cabibbo-Kobayashi-Maskawa (CKM) matrix, leading to large mixing in the 2-3 sector. It is nevertheless possible to find particular textures for $Y_{\nu}$ and $M_{N}$ for which the ratios of $\mu$ and $\tau$ decays are simultaneously closer to the experimental bounds. These cases will, however, typically imply smaller values for the Dirac Yukawa couplings of the first and second generations, predicting less restrictive BRs. Our study can be considered as targeting the kinds of textures that predict large charged LFV.

\subsection{Combining $\mu \rightarrow e \gamma$ and LHC bounds}

The scale $M_{R}=2.5 \cdot 10^{12} \mathrm{GeV}$ was chosen as representative. It also turns out that no points are excluded by $\tau \rightarrow \mu \gamma$, since $\mu \rightarrow e \gamma$ is more restrictive. This bound, in combination with large mixing for solar and atmospheric neutrinos, also excludes models with rare $\tau$ decays at the levels of the experimental limits in almost all natural textures. Keeping this in mind, we proceed to analyze the predictions for this decay in different unification schemes, studying all kinds of DM models. Since the signal largely depends on the SUSY particle spectroscopy, we combine our analysis with consideration of the LHC data for the specific unified SUSY models under consideration. For this purpose we follow a similar procedure as that applied in refs. $[57,58]$. Each model can be associated to a particular set of particle mass hierarchies and decays, which are then compared with the generic analyses provided by the ATLAS and CMS collaborations [111, 112]. These comparisons are made with the help of Simplified Model Spectra (SMS) which can be defined by a set of hypothetical SUSY particle masses and a sequence of decay patterns that have to be compared with those expected in any specific model. An individual check has to be done for every model, while, due to mismatches between the theoretical predictions and the experimental analyses, it is not possible to provide contour plots where one can easily see which mass ranges are excluded. This task is simplified by using public packages like Smodels-v1.2.2 [113], which provides a powerful tool for performing a fast analysis of a large number of models $[114,115]$. Using this package, the theoretical models are mapped onto SMS and can be compared with the existing LHC bounds if there is a match in the respective topologies. In each model the mass spectrum is generated using SoftSusy and the corresponding decay ratios are calculated using SUSY-HIT [116]. The cross-section information is then inserted in Smodels-v1.2.2 through a call to Pythia 8.2 [117].

We classify the models as follows, according to their LHC prospects:

(i) Those that are excluded by the current LHC bounds;

(ii) Those that can be compared with the LHC data and are not excluded;

(iii) Those that cannot be tested at the LHC, i.e., points that predict either processes with very low cross sections or topologies that are not tested at the LHC. 

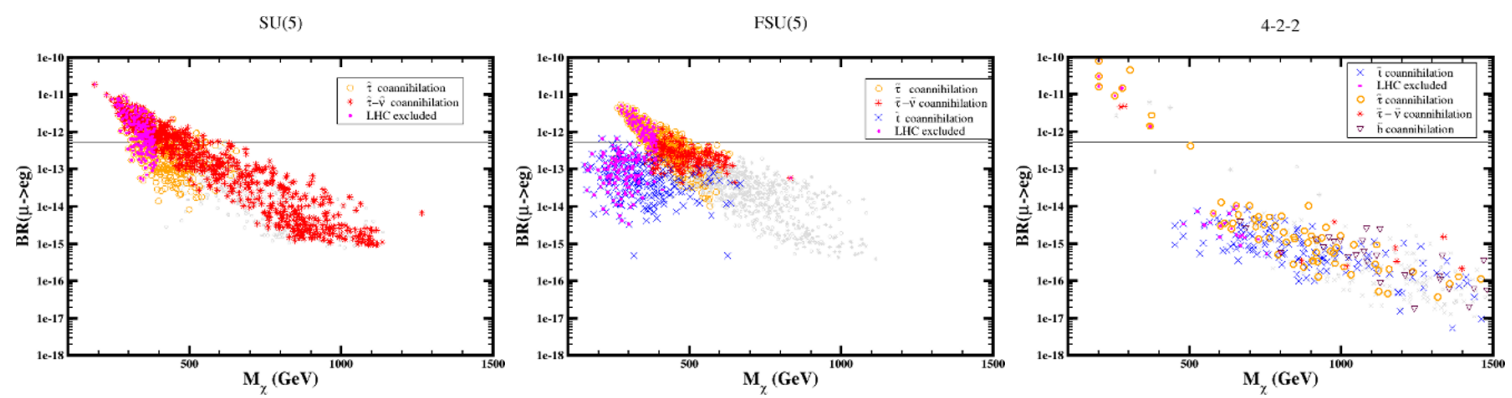

Figure 3. Prediction for $\operatorname{BR}(\mu \rightarrow e \gamma)$ vs $m_{\chi}$ for (from left to right) the $\mathrm{SU}(5)$, the $\mathrm{FSU}(5)$ and 4-2-2 models, in scenarios where sfermions coannihilate with the LSP. We use the same notation for the DM models as in figure 2. Models with parameters not detectable at the LHC are marked in grey, while excluded models are marked with purple dots. We assume $M_{N}=2.5 \cdot 10^{12} \mathrm{GeV}$ in all three cases.
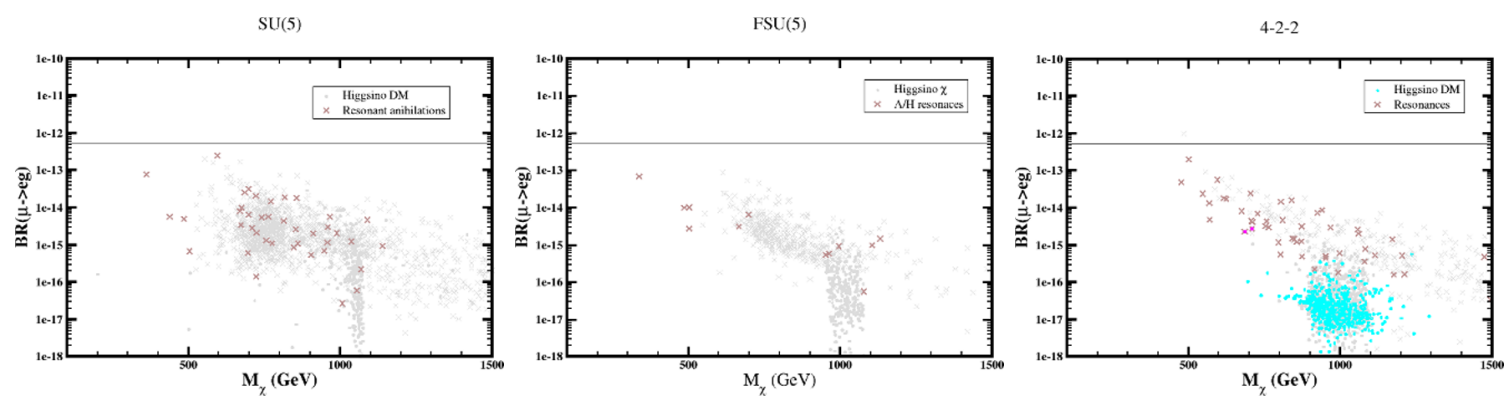

Figure 4. As in figure 3, for Higgsino DM and for models of resonant coannihilation.

In figure 3 and following figures, we denote points of the same DM class with the same symbol as in figure 2 but changing the colour according to the LHC prospects of the model: points of categories (i) and (ii) retain the same colour as in figure 2, adding a magenta dot for the excluded ones, whereas points of category (iii) are drawn in grey.

We see in figure 3 that the $\mu \rightarrow e \gamma$ bound may be violated in DM models with coannihilations, whereas models with resonant annihilations and higgsino DM are not affected by this bound, as seen in figure 4 . This can be attributed to the lighter masses of the sleptons in the coannihilation scenarios.

The LHC and charged LFV predictions of models populating classes of points with different DM mechanisms can be compared in the different unification scenarios:

$\tilde{\boldsymbol{\tau}}-\chi$ and $\tilde{\boldsymbol{\tau}}-\tilde{\boldsymbol{\nu}}-\chi$ coannihilation. These mechanisms are particularly interesting, since they both predict LFV and LHC signals within experimental reach. In the $\tilde{\tau}-\chi$ scenario, the lighter stau is determined by left-right mixing, and the $\tilde{\tau}-\tilde{\nu}-\chi$ is the limiting case where the $\tilde{\tau}_{1}$ is mainly left-handed. We should also take into account the fact that LFV is induced mainly in the left-left sector of the slepton mass matrix, due to the see-saw mechanism, therefore models with larger left-stau composition and smaller masses tend to have larger LFV decay rates. As seen in figure 4, this scenario is very interesting in SU(5) and FSU(5), since these models predict both LFV and LHC signals within experimental reach. Models with LSP masses above $400 \mathrm{GeV}$ are not excluded in 


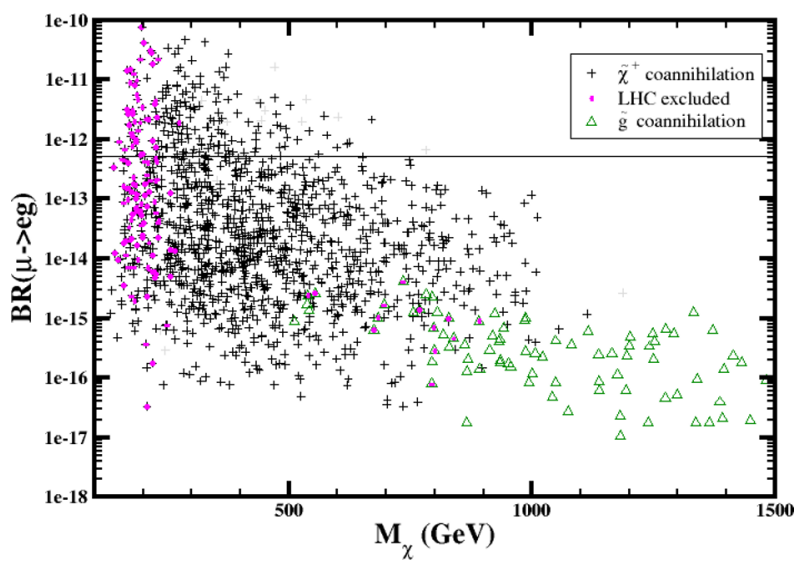

Figure 5. As in figures 3 and 4 for models with chargino and gluino coannihilations in the 4-2-2 GUT.

either scenario, but the different representation assignments and hence soft masses change the slepton compositions, with manifest implications for the LFV predictions, which are specific for each group. For instance, whereas in $\mathrm{SU}(5)$ most of the points with $\tilde{\tau}-\tilde{\nu}-\chi$ coannihilations violate the experimental bound, in $\mathrm{FSU}(5)$ they are still allowed. In the case of 4-2-2 models, there is a left-right splitting of the sfermion soft masses, implying that points with stau coannihilations are more difficult to find than in $\mathrm{SU}(5)$, as can be seen in the corresponding panel of figure 3. Moreover, due to gaugino mass relations, the charginos and neutralinos can be heavier than in $\mathrm{SU}(5)$ models, leading to lower $\mathrm{BR}(\mu \rightarrow e \gamma)$.

$\tilde{\boldsymbol{t}}-\chi$ coannihilation. Figure 4 shows that such models are present in the FSU(5) and in the 4-2-2 schemes, but the predictions are different in the two frameworks. In FSU(5), models with LSP masses up to $700 \mathrm{GeV}$ can predict ratios up to one order of magnitude below the current bound, whereas in 4-2-2 models the LSP mass can be larger, with $\operatorname{BR}(\mu \rightarrow e \gamma)$ two orders of magnitude below the experimental limit.

$\boldsymbol{A} / \boldsymbol{H}$ resonances. As can be seen in figure 4, the predictions for LFV decays are below the current limits. However, there are some differences between the three GUTs for the points with good prospects for both the $\mathrm{LHC}$ and $\mathrm{BR}(\mu \rightarrow e \gamma)$, which are easier to find in 4-2-2 and $\mathrm{SU}(5)$ than in $\mathrm{FSU}(5)$.

Higgsino DM. Figure 4 shows that this class of points does not predict charged LFV of experimental interest, due to the heavy SUSY masses in the three GUT schemes; these models are also out the LHC reach in all SU(5) cases. However, the LSP composition is different in the three schemes; for instance, in the 4-2-2 model the LSP is almost a pure Higgsino and, even if $\mathrm{BR}(\mu \rightarrow e \gamma)$ is low, some model points can be tested at the LHC.

$\tilde{\chi}^{+}-\chi$ and $\tilde{\boldsymbol{g}}-\chi$ coannihilations. These DM classes appear only in the 4-2-2 case, due to its GUT relation on gaugino masses. As can be seen in figure 5, models with $\tilde{\chi}^{+}-\chi$ coannihilations have good detection prospects for both LFV decays and at the LHC. Points with $\tilde{g}-\chi$ coannihilation are still within the $\mathrm{LHC}$ reach, while the $\mathrm{BR}(\mu \rightarrow e \gamma)$ predictions are low. 


\subsection{LFV signals, SUSY spectroscopy and DM detection}

In this section we discuss the LHC prospects for discovering SUSY combined with a possible charged LFV signal. The results are shown in figures 6 and 7, which plot SUSY particle masses vs. $m_{\chi}$, in order to compare directly the range of SUSY masses to which the LHC is sensitive with those that give rise to detectable LFV signatures. In the case of SU(5) and FSU(5), each panel contains all classes of points, while in the 4-2-2 case the different classes are shown in two panels, for clarity of presentation.

We follow the same notation as in the previous section, with purple dots denoting points excluded by the LHC. In addition to the symbols introduced in the previous sections, we introduce two more, to show the impact of the LFV predictions on the SUSY spectrum:

- Indigo crosses mark points excluded by the current bound on $\operatorname{BR}(\mu \rightarrow e \gamma)$, and

- Green crosses mark points with predictions for $\mathrm{BR}(\mu \rightarrow e \gamma)$ between the present bound and a factor of 10 below this value.

In addition, the solid red lines are obtained by combining the simplified model bounds from LHC searches. Since these bounds often do not apply directly to our particular cases, this boundary should not be considered as an exclusion line, though excluded points would lie within at least one of these contours. Nevertheless, it is useful to include this line for illustrative purposes, since it gives an idea of the range of masses explored at the LHC for every SUSY particle.

The upper panels in figure 6 and 7 display LHC and LFV results on $m_{\tilde{g}}-m_{\chi}$ contour plots. Since in SU(5) and FSU(5) we assume universal gaugino masses at the GUT scale, all except the Higgsino DM models lie on the proportionality lines obtained from the GUT relations. Among other relations, the neutralino mass is in general proportional to that of the gluino, something that does not hold in 4-2-2 where, due to its different group structure, the distribution of models (shown in figure 7), follows different patterns. The sfermion coannihilation cases (left panel) do not show any correlation in the $m_{\tilde{g}}-m_{\chi}$ plane. The same holds for models with Higgsino DM and with A/H resonances (right panel), which deviate from the proportionality line. Chargino and gluino coannihiliations, on the other hand, display the pattern of mass correlations described in ref. [57]. We have checked that the excluded points inside the red contour in $m_{\tilde{g}}-m_{\chi}$ plots in figures 6 and 7 violate the constraint from the 0 -lepton + jets $+E_{T}$ channel $[118,119]$. This bound affects all the models excluded by the LHC in $\mathrm{SU}(5)$, and most of the models excluded in the other two scenarios.

Although the superposition of models on figures 6 and 7 does not by itself allow a clear distinction among different DM scenarios, we can associate the excluded points to specific models by confronting these figures with the LFV predictions of figures 3,4 and 5 . We see that models with sfermion coannihilations in SU(5) and FSU(5) are more affected by the LHC bounds than in the 4-2-2 case, especially for $\tilde{t}-\chi$ coannihilations. In all scenarios, LFV enables exploring a range of $m_{\tilde{g}}$ far beyond the LHC bounds (up to about $4 \mathrm{TeV}$ in $\mathrm{SU}(5)$ and FSU(5), and even larger values in 4-2-2 in the chargino coannihilation scenario).

The analysis of excluded models shown in the $m_{\tilde{t}}-m_{\chi}$ plots (middle panels of figures 6 and 7 ) indicates that they are affected by the bound due to searches for stop decays into 
$\mathrm{SU}(5)$
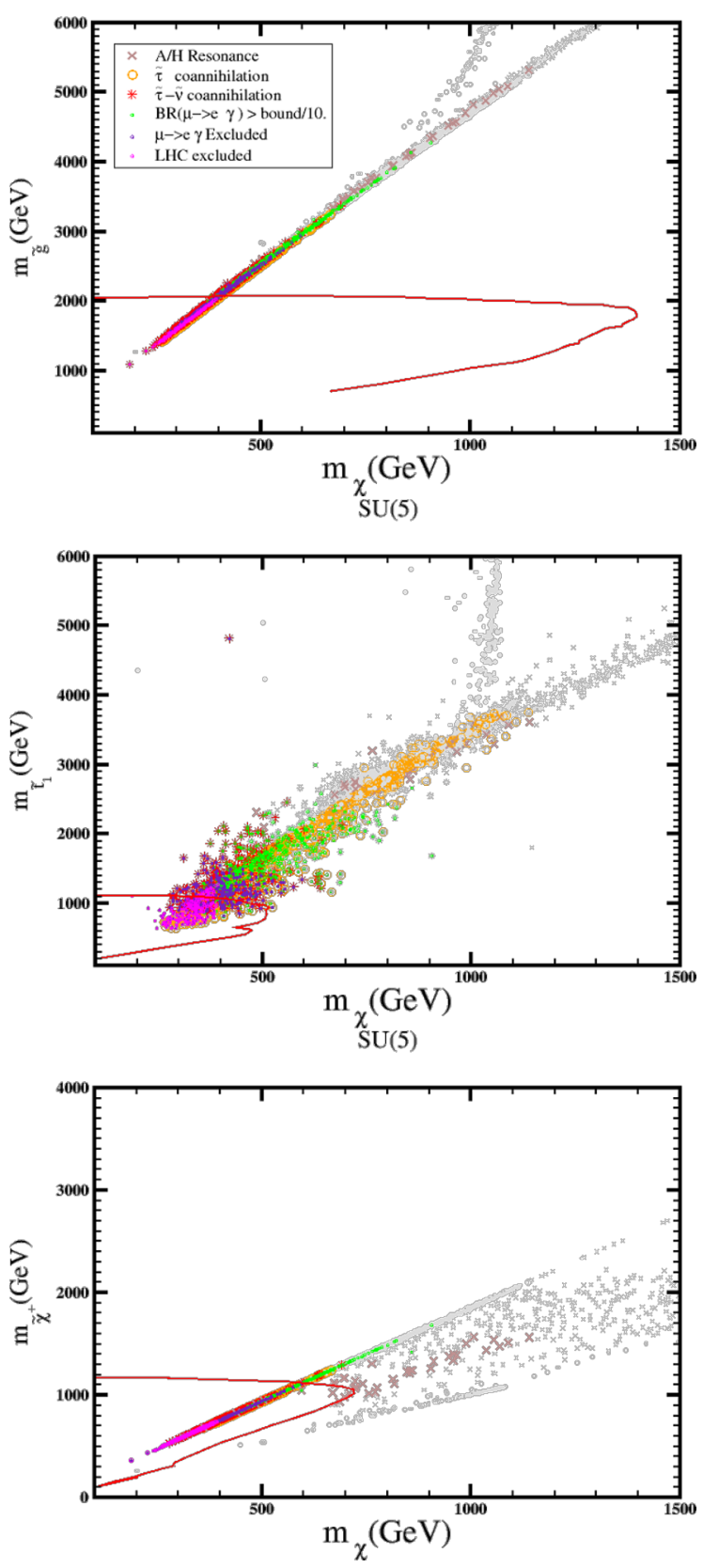

FSU(5)
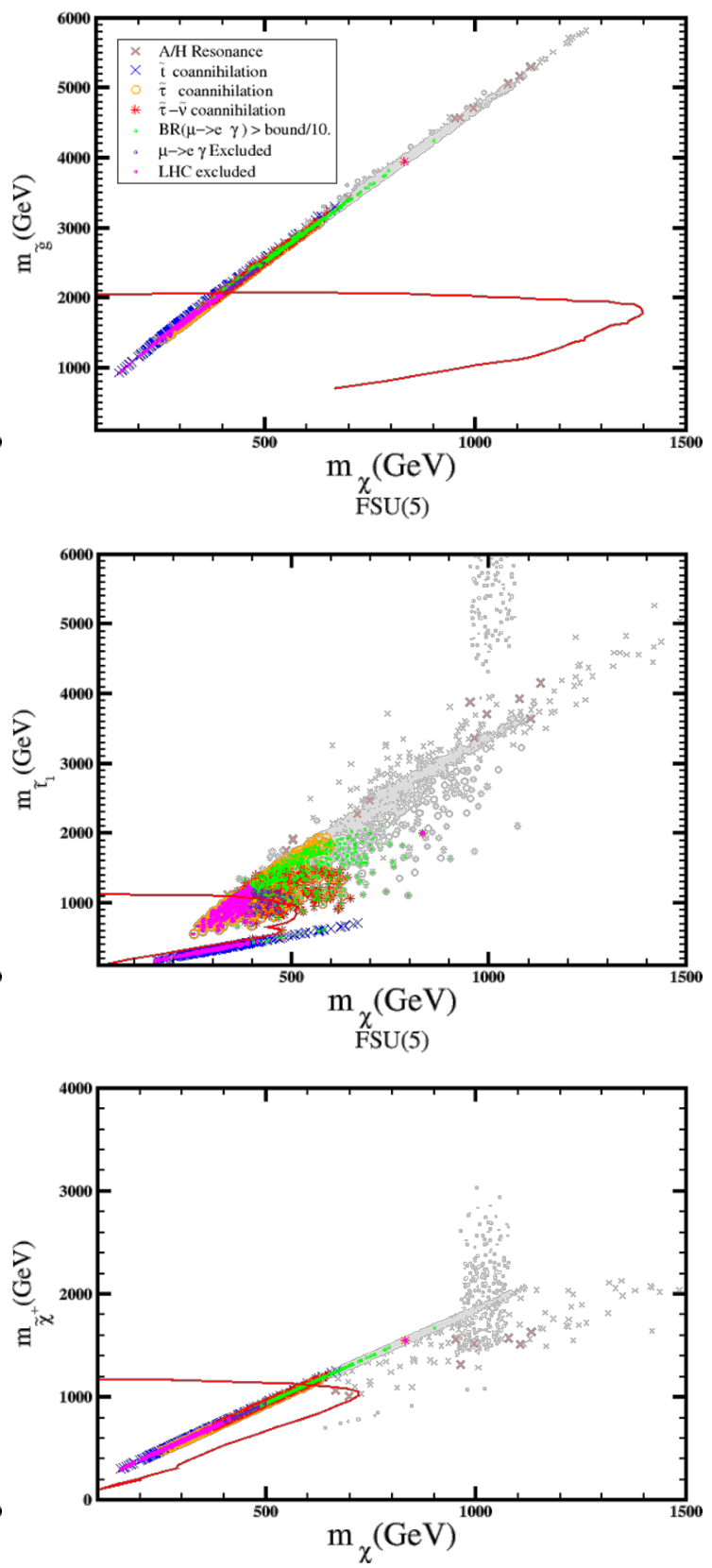

Figure 6. LHC prospects for the SU5 and FSU5 models. The points follow the notation of figures 3, 4 and 5. The meanings of the solid red lines are explained in the text. Indigo crosses indicate points excluded by the limit on $\operatorname{BR}(\mu \rightarrow e \gamma)$, whereas the green crosses mark points that lie between the current bound and one order of magnitude below it. 
4-2-2
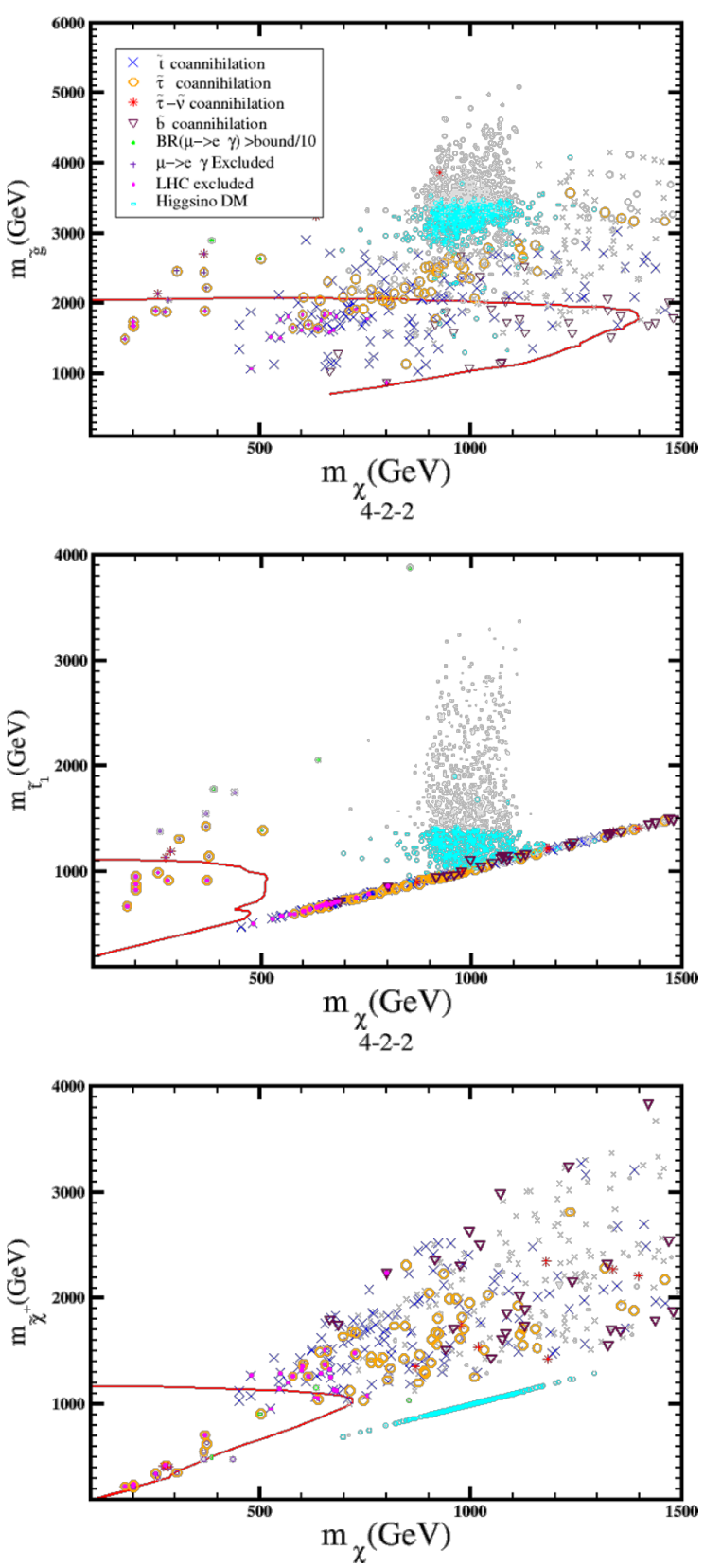

4-2-2
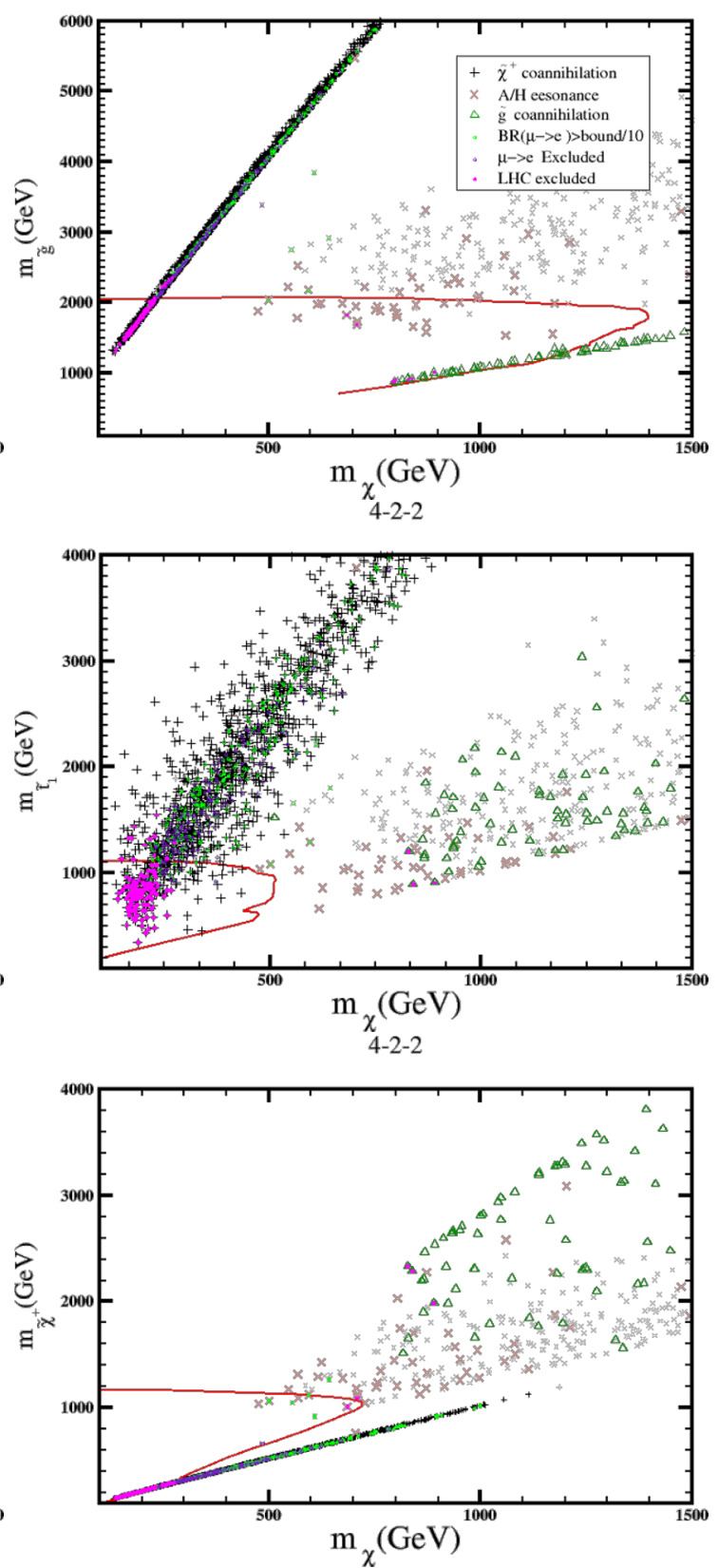

Figure 7. LHC prospects for the 4-2-2 model, following the notations of figure 6. For clarity of presentation, in the left panels we display predictions for models with sfermion coannihilations, whereas in the right panels we display the remaining cases. 

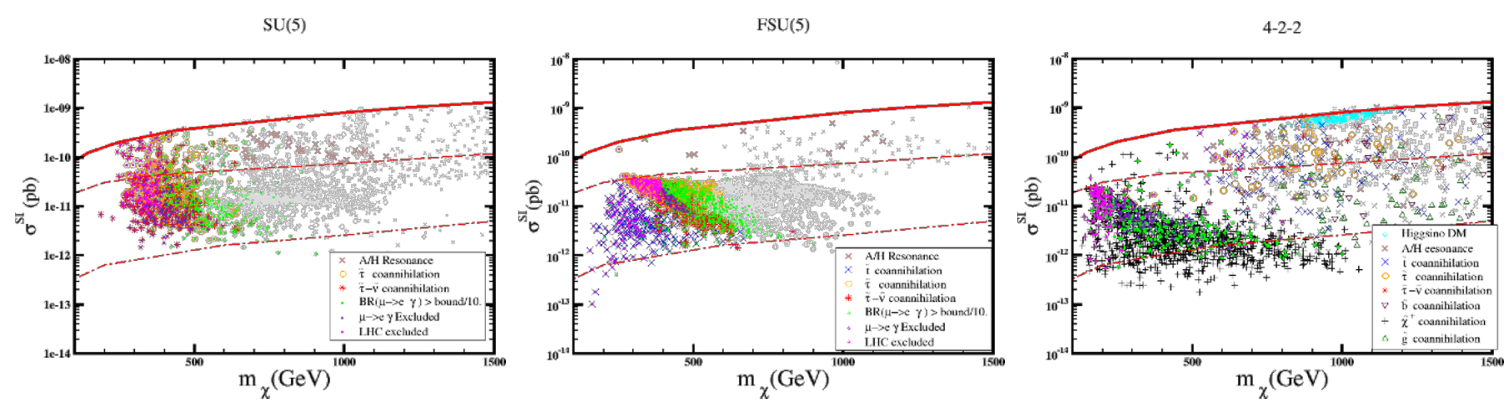

Figure 8. SI neutralino-nucleon cross section versus $m_{\chi}$ in SU(5), FSU(5) and the 4-2-2 escenarios. The solid lines corresponds to the Xenon-1T bound [11], and the dashed and dot-dashed lines correspond to the projected sensitivities of the LZ [125] and DARWIN [126] experiments.

$t-\chi^{ \pm}[120-123]$. We see that the exclusion bound in the SU(5) and FSU(5) panels contains many points with slepton coannihilations, while points with $\tilde{t}-\chi$ coannihilations escape this bound. In the case of the 4-2-2 models shown in the left middle panel of figure 7 , we see that this bound is less effective for the same kind of models than in the other GUTs. In the middle right panel of the same figure, we see that the bound excludes many models with $\tilde{\chi}^{ \pm}-\chi$ coannihilations with $m_{\chi}$ below $300 \mathrm{GeV}$. Regarding LFV, we see that the models present good detection prospects up to stop masses above $3 \mathrm{TeV}$ (and even further in 4-2-2 models). However, in the case of 4-2-2, only models with $\tilde{\chi}^{+}-\chi$ coannihilation predict LFV within one order of magnitude of the current bound.

The $m_{\tilde{\chi}^{ \pm}}-m_{\chi}$ plane (bottom panels of figures 6 and 7 ) shows that it is possible to see models excluded by electroweak searches through the ATLAS multi-leptons $+\mathscr{E}_{T}$ channel [124]. This channel is particularly important in models with $\tilde{\chi}^{ \pm}-\chi$ coannihilation in the 4-2-2 scenario, where it can exclude models allowed by 0 -lepton + jets $+\mathscr{E}_{T}$. We see that most of the models with chargino mass $m_{\chi^{ \pm}} \lesssim 300 \mathrm{GeV}$ are excluded by these searches. Regarding LFV in SU(5) and FSU(5), we see that the models give rise to good detection prospects for chargino masses up to $1.5 \mathrm{TeV}$ whilst, in the case of 4-2-2, only models with chargino coannihilation present better prospects for LFV detection. In these cases, the masses reach the maximum value of $1 \mathrm{TeV}$ within our data range. The impact is weaker for sbottom searches, as was shown in $[57,58]$. This is due to the fact that in our scenarios the sbottom squarks are heavy and outside the area covered by the LHC; the same happens with signals involving squarks of the lighter generations.

Finally, we display in figure 8 the spin-independent (SI) neutralino-nucleon cross section as a function of the neutralino mass in the different GUT models, and we see that the predictions depend on the unification scenario. We note in particular that the FSU(5) model predicts a lower SI cross section than the SU(5) model, in general, while the 42-2 model may yield a relatively large SI cross section even for large neutralino masses $>1 \mathrm{TeV}$. The current bound from the Xenon-1T experiment [11] already excludes many models where the neutralino has a large higgsino component, and the projected sensitivities of the LZ [125] and DARWIN [126] experiments will be able to cover most of the models studied on this work. In particular, only models with $\tilde{\chi}^{ \pm}-\chi$ coannihilations may escape the projected DARWIN sensitivity. Comparing the sensitivities of the LFV, LHC 
and SI DM searches, we see that the latter are potentially very promising probes of SUSY models. On the other hand, as in [57], we find in each model that the spin-dependent (SD) neutralino-neutron cross section is below the projected limit from the LZ [125] experiment.

\section{Conclusions}

In previous work, we studied the predictions of different unified theories for DM and the LHC. We investigated several GUT scenarios, comparing the areas allowed within different symmetry schemes. We considered scenarios with gaugino unification, such as $\mathrm{SU}(5)$ and FSU(5), and models where it can be relaxed, such as 4-2-2 models. Among others, we had reached the following conclusions:

- Models based on $\mathrm{SO}(10)$ are very restricted by data, as can be seen in refs. [5658]. In contrast, SU(5) models contain several areas of interest for higgsino dark matter, resonant annihilations and coannihilations. However, due to its multiplet structure, SU(5) models not allow stop-neutralino coannihilations, in contrast to the other groups.

- Flipped SU(5) models can be clearly distinguished from SU(5), and have several additional features, including stop-neutralino coannihilations.

- Models based on 4-2-2 not only give rise to stop-neutralino and sbottom-neutralino coannihilations, they also allow novel DM mechanisms, including gluino and chargino coannihilations, as a direct consequence of the distinctive gauge structure.

Here we have combined these analyses with the study of LFV, which turns out to be particularly relevant, using updated LHC data. The large mixing for solar and atmospheric neutrinos implies strong correlations between different rare decays. Since the limits for $\mu \rightarrow e \gamma$ are significantly stronger, it made sense to focus mostly on this mode and comment on $\tau \rightarrow \mu \gamma$ where relevant. We have found the following:

- The three groups have distinctive LFV signatures, making it possible to link specific signatures in rare decays and colliders to the gauge and multiplet structure of the theory.

- The results are naturally sensitive to the scale of the right-handed neutrinos, $M_{R}$. The see-saw mechanism implies that larger scales are linked to larger couplings and thus larger quantum corrections that violate flavour. For smaller values of $M_{R}$ the available parameter space is significantly enhanced: indeed, a change of $M_{R}$ by a factor of 4 is sufficient to exclude or allow a large number of models.

- In all three groups, coannihilations lead to higher rates for LFV, while resonant annihilations and higgsino dark matter are mostly not affected. Overall, in SU(5) and 4-2-2 it is easier to find annihilation models with good detection prospects both at the LHC and in LFV searches. Higgsino DM models do not predict detectable LFV. Still, it is interesting to note that the LSP composition is different in each scheme, yielding an almost pure Higgsino spectrum in the 4-2-2 model. 
- Since the see-saw mechanism introduces LFV only in the LL sector, stau coannihilations with smaller masses and larger left-stau components lead to LFV within the current reach. This is particularly relevant for SU(5) and flipped $\mathrm{SU}(5)$, since in 4-2-2 models the left-right splitting of the soft fermion masses makes stau coannihilations more difficult to find. However, the two groups can be clearly distinguished, since $\mathrm{SU}(5)$ is more restrictive than flipped $\mathrm{SU}(5)$.

- Stop-neutralino coannihilations appear only in flipped SU(5) and 4-2-2 models but, once more, with distinct signatures in each case. In 4-2-2 models the LSP can be heavier, and significantly smaller LFV rates are to be expected.

- The 4-2-2 model also allows chargino and gluino coannihilations with neutralinos, due to the different GUT relations for gaugino masses. Chargino-neutralino coannihilations have good detection prospects for both the LHC and LFV, while gluino coannihilations lead to lower LFV rates.

- There are specific correlations between the sparticle masses, leading to interesting signatures. In flipped $\mathrm{SU}(5)$, gaugino mass universality results in a proportionality between the gluino and neutralino masses for most of the models under study (corresponding to Higgsino DM and resonant annihilations). Larger masses have good LFV detection prospects, even when they are out of the LHC reach. This is also true for stop-neutralino coannihilations, as well as for models with compressed spectra, such as stau coannihilations.

- In 4-2-2 models, a proportionality relation is found only in chargino-neutralino coannihilations, again due to the GUT relation when the chargino is mostly a Wino. This class of models provides good prospects for both LFV and the LHC, while in other scenarios LFV is significant only for neutralino masses below $500 \mathrm{GeV}$. This is an additional feature that enables detailed tests of neutralino-chargino coannihilations versus alternative possibilities.

- The experimental advances in direct LSP DM detection are already reaching the sensitivity needed to provide a verdict on many models, specially on the $\mathrm{SU}(5)$ and 4-2-2 GUTs. Also, the projected sensitivities of the LZ and DARWIN experiments will provide probes of models that are complementary to LFV searches, even models that cannot be explored at the LHC.

Overall, our results indicate that LFV is a powerful tool that complements LHC and DM searches, and provides valuable information that can help identify optimal modes for future LHC searches. Moreover, not only does it distinguish clearly between various GUTs via the observability of different channels, but it can also provide significant insight into the respective sparticle spectra and neutrino mass parameters. 


\section{Acknowledgments}

The work of J.E. was supported by the UK STFC Grant ST/P000258/1 and by the Estonian Research Council via a Mobilitas Pluss grant. The research of M.E.G. was supported by the Spanish MINECO, under grant FPA2017-86380. R. RdA acknowledges partial funding/support from the Elusives ITN (Marie Skłodowska-Curie grant agreement No 674896) and the "SOM Sabor y origen de la Materia" (FPA 2017-85985-P). Q.S. acknowledges support by the US DOE grant No. DE-SC0013880.

Open Access. This article is distributed under the terms of the Creative Commons Attribution License (CC-BY 4.0), which permits any use, distribution and reproduction in any medium, provided the original author(s) and source are credited.

\section{References}

[1] WMAP collaboration, Seven-Year Wilkinson Microwave Anisotropy Probe (WMAP) Observations: Cosmological Interpretation, Astrophys. J. Suppl. 192 (2011) 18 [arXiv: 1001.4538] [INSPIRE].

[2] WMAP collaboration, Nine-Year Wilkinson Microwave Anisotropy Probe (WMAP) Observations: Final Maps and Results, Astrophys. J. Suppl. 208 (2013) 20 [arXiv: 1212.5225] [INSPIRE].

[3] Planck collaboration, Planck 2013 results. XVI. Cosmological parameters, Astron. Astrophys. 571 (2014) A16 [arXiv:1303.5076] [INSPIRE].

[4] Planck collaboration, Planck 2015 results. XIII. Cosmological parameters, Astron. Astrophys. 594 (2016) A13 [arXiv:1502.01589] [INSPIRE].

[5] ATLAS collaboration, Observation of a new particle in the search for the Standard Model Higgs boson with the ATLAS detector at the LHC, Phys. Lett. B 716 (2012) 1 [arXiv:1207.7214] [INSPIRE].

[6] CMS collaboration, Observation of a New Boson at a Mass of $125 \mathrm{GeV}$ with the CMS Experiment at the LHC, Phys. Lett. B 716 (2012) 30 [arXiv:1207.7235] [INSPIRE].

[7] https://twiki.cern.ch/twiki/bin/view/CMSPublic/PhysicsResultsSUS.

[8] https://twiki.cern.ch/twiki/bin/view/AtlasPublic/SupersymmetryPublicResults.

[9] LUX collaboration, Results from a search for dark matter in the complete LUX exposure, Phys. Rev. Lett. 118 (2017) 021303 [arXiv: 1608.07648] [INSPIRE].

[10] XENON collaboration, First Dark Matter Search Results from the XENON1T Experiment, Phys. Rev. Lett. 119 (2017) 181301 [arXiv:1705.06655] [INSPIRE].

[11] XENON collaboration, Dark Matter Search Results from a One Ton-Year Exposure of XENON1T, Phys. Rev. Lett. 121 (2018) 111302 [arXiv:1805.12562] [INSPIRE].

[12] PandaX-II collaboration, Dark Matter Results From 54-Ton-Day Exposure of PandaX-II Experiment, Phys. Rev. Lett. 119 (2017) 181302 [arXiv:1708. 06917] [INSPIRE].

[13] PICO collaboration, Dark Matter Search Results from the PICO-60 $C_{3} F_{8}$ Bubble Chamber, Phys. Rev. Lett. 118 (2017) 251301 [arXiv:1702. 07666] [INSPIRE]. 
[14] H. Goldberg, Constraint on the Photino Mass from Cosmology, Phys. Rev. Lett. 50 (1983) 1419 [Erratum ibid. 103 (2009) 099905] [INSPIRE].

[15] J.R. Ellis, J.S. Hagelin, D.V. Nanopoulos, K.A. Olive and M. Srednicki, Supersymmetric Relics from the Big Bang, Nucl. Phys. B 238 (1984) 453 [inSPIRE].

[16] J.R. Ellis, S. Kelley and D.V. Nanopoulos, Precision LEP data, supersymmetric GUTs and string unification, Phys. Lett. B 249 (1990) 441 [INSPIRE].

[17] J.R. Ellis, S. Kelley and D.V. Nanopoulos, Probing the desert using gauge coupling unification, Phys. Lett. B 260 (1991) 131 [INSPIRE].

[18] U. Amaldi, W. de Boer and H. Furstenau, Comparison of grand unified theories with electroweak and strong coupling constants measured at LEP, Phys. Lett. B 260 (1991) 447 [INSPIRE].

[19] P. Langacker and M.-x. Luo, Implications of precision electroweak experiments for $M_{t}, \rho_{0}$, $\sin ^{2} \theta_{W}$ and grand unification, Phys. Rev. D 44 (1991) 817 [INSPIRE].

[20] C. Giunti, C.W. Kim and U.W. Lee, Running coupling constants and grand unification models, Mod. Phys. Lett. A 6 (1991) 1745 [InSPIRE].

[21] F. Borzumati and A. Masiero, Large Muon and electron Number Violations in Supergravity Theories, Phys. Rev. Lett. 57 (1986) 961 [InSPIRE].

[22] MEG collaboration, New constraint on the existence of the $\mu^{+} \rightarrow e^{+} \gamma$ decay, Phys. Rev. Lett. 110 (2013) 201801 [arXiv:1303.0754] [INSPIRE].

[23] Particle Data Group collaboration, Review of Particle Physics, Chin. Phys. C 38 (2014) 090001 [INSPIRE].

[24] I.-H. Lee, Lepton Number Violation in Softly Broken Supersymmetry, Phys. Lett. B 138 (1984) 121 [INSPIRE].

[25] I.-H. Lee, Lepton Number Violation in Softly Broken Supersymmetry. 2., Nucl. Phys. B 246 (1984) 120 [INSPIRE].

[26] N.V. Krasnikov, Search for flavor lepton number violation in slepton decays at LHC, JETP Lett. 65 (1997) 148 [hep-ph/9611282] [INSPIRE].

[27] S.I. Bityukov and N.V. Krasnikov, The Search for charged sleptons and flavor lepton number violation at LHC (CMS), in 10th International Seminar on High-Energy Physics (Quarks 98), (1998) [hep-ph/9806504] [INSPIRE].

[28] K. Agashe and M. Graesser, Signals of supersymmetric lepton flavor violation at the CERN LHC, Phys. Rev. D 61 (2000) 075008 [hep-ph/9904422] [INSPIRE].

[29] I. Hinchliffe and F.E. Paige, Lepton flavor violation at the CERN LHC, Phys. Rev. D 63 (2001) 115006 [hep-ph/0010086] [INSPIRE].

[30] J. Hisano, R. Kitano and M.M. Nojiri, Slepton oscillation at large hadron collider, Phys. Rev. D 65 (2002) 116002 [hep-ph/0202129] [INSPIRE].

[31] D.F. Carvalho, J.R. Ellis, M.E. Gómez, S. Lola and J.C. Romão, Tau flavor violation in sparticle decays at the LHC, Phys. Lett. B 618 (2005) 162 [hep-ph/0206148] [INSPIRE].

[32] A. Bartl, K. Hidaka, K. Hohenwarter-Sodek, T. Kernreiter, W. Majerotto and W. Porod, Test of lepton flavor violation at LHC, Eur. Phys. J. C 46 (2006) 783 [hep-ph/0510074] [INSPIRE]. 
[33] E. Carquin, J. Ellis, M.E. Gómez, S. Lola and J. Rodríguez-Quintero, Search for Tau Flavour Violation at the LHC, JHEP 05 (2009) 026 [arXiv: 0812.4243] [INSPIRE].

[34] A. Abada, A.J.R. Figueiredo, J.C. Romão and A.M. Teixeira, Probing the supersymmetric type-III seesaw: LFV at low-energies and at the LHC, JHEP 08 (2011) 099 [arXiv:1104.3962] [INSPIRE].

[35] J.N. Esteves, J.C. Romão, A. Villanova del Moral, M. Hirsch, J.W.F. Valle and W. Porod, Flavour violation at the LHC: type-I versus type-II seesaw in minimal supergravity, JHEP 05 (2009) 003 [arXiv:0903.1408] [INSPIRE].

[36] M. Hirsch, W. Porod, C. Weiss and F. Staub, Supersymmetric type-III seesaw mechanism: Lepton flavor violation and LHC phenomenology, Phys. Rev. D 87 (2013) 013010 [arXiv: 1211.0289] [INSPIRE].

[37] N. Arkani-Hamed, H.-C. Cheng, J.L. Feng and L.J. Hall, Probing lepton flavor violation at future colliders, Phys. Rev. Lett. 77 (1996) 1937 [hep-ph/9603431] [INSPIRE].

[38] N. Arkani-Hamed, J.L. Feng, L.J. Hall and H.-C. Cheng, CP violation from slepton oscillations at the LHC and NLC, Nucl. Phys. B 505 (1997) 3 [hep-ph/9704205] [INSPIRE].

[39] J. Hisano, M.M. Nojiri, Y. Shimizu and M. Tanaka, Lepton flavor violation in the left-handed slepton production at future lepton colliders, Phys. Rev. D 60 (1999) 055008 [hep-ph/9808410] [INSPIRE].

[40] M. Guchait, J. Kalinowski and P. Roy, Supersymmetric lepton flavor violation in a linear collider: The Role of charginos, hep-ph/0103161 [INSPIRE].

[41] F. Deppisch, J. Kalinowski, H. Päs, A. Redelbach and R. Rückl, Supersymmetric lepton flavor violation at the $L H C$ and $L C$, hep-ph/0401243 [INSPIRE].

[42] F. Deppisch, H. Päs, A. Redelbach, R. Rückl and Y. Shimizu, The SUSY seesaw model and lepton flavor violation at a future electron positron linear collider, Phys. Rev. D 69 (2004) 054014 [hep-ph/0310053] [INSPIRE].

[43] M. Cannoni, C. Carimalo, W. Da Silva and O. Panella, Testing SUSY models of lepton flavor violation at a photon collider, Phys. Rev. D 72 (2005) 115004 [Erratum ibid. 72 (2005) 119907] [hep-ph/0508256] [INSPIRE].

[44] E. Carquin, J. Ellis, M.E. Gómez and S. Lolab, Searches for Lepton Flavour Violation at a Linear Collider, JHEP 11 (2011) 050 [arXiv:1106.4903] [INSPIRE].

[45] A. Abada, A.J.R. Figueiredo, J.C. Romão and A.M. Teixeira, Lepton flavour violation: physics potential of a Linear Collider, JHEP 08 (2012) 138 [arXiv:1206.2306] [INSPIRE].

[46] J.C. Pati and A. Salam, Lepton Number as the Fourth Color, Phys. Rev. D 10 (1974) 275 [Erratum ibid. 11 (1975) 703] [INSPIRE].

[47] R.N. Mohapatra and J.C. Pati, Left-Right Gauge Symmetry and an Isoconjugate Model of CP-violation, Phys. Rev. D 11 (1975) 566 [InSPIRE].

[48] G. Senjanović and R.N. Mohapatra, Exact Left-Right Symmetry and Spontaneous Violation of Parity, Phys. Rev. D 12 (1975) 1502 [inSPIRE].

[49] M. Magg, Q. Shafi and C. Wetterich, Gauge Hierarchy in Presence of Discrete Symmetry, Phys. Lett. B 87 (1979) 227 [InSPIRE].

[50] G. Lazarides and Q. Shafi, Comments on 'Monopole Charges in Unified Gauge Theories', Nucl. Phys. B 189 (1981) 393 [INSPIRE]. 
[51] T.W.B. Kibble, G. Lazarides and Q. Shafi, Strings in SO(10), Phys. Lett. B 113 (1982) 237 [INSPIRE].

[52] M. Cannoni, J. Ellis, M.E. Gómez, S. Lola and R. Ruiz de Austri, Supersymmetry Searches in GUT Models with Non-Universal Scalar Masses, JCAP 03 (2016) 041 [arXiv:1511.06205] [INSPIRE].

[53] N. Okada, S. Raza and Q. Shafi, Particle Spectroscopy of Supersymmetric SU(5) in Light of 125 GeV Higgs and Muon g - 2 Data, Phys. Rev. D 90 (2014) 015020 [arXiv:1307.0461] [INSPIRE].

[54] K. Kowalska, L. Roszkowski, E.M. Sessolo and A.J. Williams, GUT-inspired SUSY and the muon g- 2 anomaly: prospects for LHC 14 TeV, JHEP 06 (2015) 020 [arXiv:1503.08219] [INSPIRE].

[55] K. Kowalska, L. Roszkowski, E.M. Sessolo and S. Trojanowski, Low fine tuning in the MSSM with higgsino dark matter and unification constraints, JHEP 04 (2014) 166 [arXiv: 1402.1328] [INSPIRE].

[56] J. Ellis, J.L. Evans, A. Mustafayev, N. Nagata and K.A. Olive, The Super-GUT CMSSM Revisited, Eur. Phys. J. C 76 (2016) 592 [arXiv: 1608.05370] [InSPIRE].

[57] M.E. Gómez, S. Lola, R. Ruiz de Austri and Q. Shafi, Confronting SUSY GUT with Dark Matter, Sparticle Spectroscopy and Muon $(g-2)$, Front. in Phys. 6 (2018) 127 [arXiv: 1806.11152] [INSPIRE].

[58] M.E. Gómez, S. Lola, R. Ruiz De Austri and Q. Shafi, Dark matter, sparticle spectroscopy and muon $(g-2)$ in $\mathrm{SU}(4)_{c} \times \mathrm{SU}(2)_{L} \times \mathrm{SU}(2)_{R}, J H E P 10$ (2018) 062 [arXiv:1806.06220] [INSPIRE].

[59] L. Roszkowski, E.M. Sessolo and A.J. Williams, What next for the CMSSM and the NUHM: Improved prospects for superpartner and dark matter detection, JHEP 08 (2014) 067 [arXiv: 1405.4289] [INSPIRE].

[60] I. Gogoladze, S. Raza and Q. Shafi, Neutralino-Sbottom Coannihilation in SU(5), JHEP 03 (2012) 054 [arXiv:1111.6299] [INSPIRE].

[61] L. Calibbi and G. Signorelli, Charged Lepton Flavour Violation: An Experimental and Theoretical Introduction, Riv. Nuovo Cim. 41 (2018) 71 [arXiv:1709.00294] [INSPIRE].

[62] A. Vicente, Lepton flavor violation beyond the MSSM, Adv. High Energy Phys. 2015 (2015) 686572 [arXiv: 1503.08622] [INSPIRE].

[63] P. Minkowski, $\mu \rightarrow$ er at a Rate of One Out of $10^{9}$ Muon Decays?, Phys. Lett. B 67 (1977) 421 [INSPIRE].

[64] M. Gell-Mann, P. Ramond and R. Slansky, Complex Spinors and Unified Theories, in Supergravity, P. Van. Nieuwenhuizen and D. Freedman eds., North-Holland, Amsterdam (1979) [Conf. Proc. C $\mathbf{7 9 0 9 2 7 ~ ( 1 9 7 9 ) ~ 3 1 5 ] ~ [ a r X i v : ~ 1 3 0 6 . 4 6 6 9 ] ~ [ I N S P I R E ] . ~}$

[65] T. Yanagida, Horizontal gauge symmetry and masses of neutrinos, in proceedings of Workshop on the Unified Theories and the Baryon Number in the Universe, O. Sawada and A. Sugamoto eds., KEK, Tsukuba (1979) [Conf. Proc. C 7902131 (1979) 95] [INSPIRE].

[66] S.L. Glashow, The Future of Elementary Particle Physics, in Quarks and Leptons, M. Lévy et al. eds., Plenum Press, New York (1980) [NATO Sci. Ser. B 61 (1980) 687] [InSPIRE].

[67] R.N. Mohapatra and G. Senjanović, Neutrino Mass and Spontaneous Parity Nonconservation, Phys. Rev. Lett. 44 (1980) 912 [INSPIRE]. 
[68] J. Schechter and J.W.F. Valle, Neutrino Masses in $\mathrm{SU}(2) \times \mathrm{U}(1)$ Theories, Phys. Rev. D 22 (1980) 2227 [INSPIRE].

[69] J. Schechter and J.W.F. Valle, Neutrino Decay and Spontaneous Violation of Lepton Number, Phys. Rev. D 25 (1982) 774 [InSPIRE].

[70] G. Lazarides, Q. Shafi and C. Wetterich, Proton Lifetime and Fermion Masses in an SO(10) Model, Nucl. Phys. B 181 (1981) 287 [InSPIRE].

[71] R.N. Mohapatra and G. Senjanović, Neutrino Masses and Mixings in Gauge Models with Spontaneous Parity Violation, Phys. Rev. D 23 (1981) 165 [inSPIRE].

[72] R. Foot, H. Lew, X.G. He and G.C. Joshi, Seesaw Neutrino Masses Induced by a Triplet of Leptons, Z. Phys. C 44 (1989) 441 [inSPIRE].

[73] J.N. Esteves, J.C. Romao, M. Hirsch, A. Vicente, W. Porod and F. Staub, LHC and lepton flavour violation phenomenology of a left-right extension of the MSSM, JHEP 12 (2010) 077 [arXiv: 1011.0348] [INSPIRE].

[74] J.N. Esteves, J.C. Romao, M. Hirsch, W. Porod, F. Staub and A. Vicente, Dark matter and LHC phenomenology in a left-right supersymmetric model, JHEP 01 (2012) 095 [arXiv: 1109.6478] [INSPIRE].

[75] Super-Kamiokande collaboration, Constraints on neutrino oscillations using 1258 days of Super-Kamiokande solar neutrino data, Phys. Rev. Lett. 86 (2001) 5656 [hep-ex/0103033] [INSPIRE].

[76] Super-Kamiokande collaboration, Solar B-8 and hep neutrino measurements from 1258 days of Super-Kamiokande data, Phys. Rev. Lett. 86 (2001) 5651 [hep-ex/0103032] [INSPIRE].

[77] Super-Kamiokande collaboration, Determination of solar neutrino oscillation parameters using 1496 days of Super-Kamiokande I data, Phys. Lett. B 539 (2002) 179 [hep-ex/0205075] [INSPIRE].

[78] CHOOZ collaboration, Limits on neutrino oscillations from the CHOOZ experiment, Phys. Lett. B 466 (1999) 415 [hep-ex/9907037] [INSPIRE].

[79] SNO collaboration, Measurement of the rate of $\nu_{e}+d \rightarrow p+p+e^{-}$interactions produced by ${ }^{8} B$ solar neutrinos at the Sudbury Neutrino Observatory, Phys. Rev. Lett. 87 (2001) 071301 [nucl-ex/0106015] [INSPIRE].

[80] SNO collaboration, Direct evidence for neutrino flavor transformation from neutral current interactions in the Sudbury Neutrino Observatory, Phys. Rev. Lett. 89 (2002) 011301 [nucl-ex/0204008] [INSPIRE].

[81] MACRO collaboration, Matter effects in upward going muons and sterile neutrino oscillations, Phys. Lett. B 517 (2001) 59 [hep-ex/0106049] [INSPIRE].

[82] MACRO collaboration, Atmospheric neutrino oscillations in MACRO, in NO-VE International Workshop on Neutrino Oscillations in Venice, pp. 207-220 (2001) [hep-ex/0110021] [INSPIRE].

[83] KAMLAND collaboration, First results from KamLAND: Evidence for reactor anti-neutrino disappearance, Phys. Rev. Lett. 90 (2003) 021802 [hep-ex/0212021] [INSPIRE].

[84] M. Cannoni, J. Ellis, M.E. Gómez and S. Lola, Neutrino textures and charged lepton flavour violation in light of $\theta_{13}, M E G$ and LHC data, Phys. Rev. D 88 (2013) 075005 [arXiv: 1301.6002] [INSPIRE]. 
[85] M.E. Gómez, G.K. Leontaris, S. Lola and J.D. Vergados, U(1) textures and lepton flavor violation, Phys. Rev. D 59 (1999) 116009 [hep-ph/9810291] [INSPIRE].

[86] J.R. Ellis, M.E. Gómez, G.K. Leontaris, S. Lola and D.V. Nanopoulos, Charged lepton flavor violation in the light of the Super-Kamiokande data, Eur. Phys. J. C 14 (2000) 319 [hep-ph/9911459] [INSPIRE].

[87] S. Antusch, E. Arganda, M.J. Herrero and A.M. Teixeira, Impact of $\theta_{13}$ on lepton flavour violating processes within SUSY seesaw, JHEP 11 (2006) 090 [hep-ph/0607263] [INSPIRE].

[88] J.R. Ellis, M.E. Gómez and S. Lola, CP and Lepton-Number Violation in GUT Neutrino Models with Abelian Flavour Symmetries, JHEP 07 (2007) 052 [hep-ph/0612292] [INSPIRE].

[89] J.A. Casas and A. Ibarra, Oscillating neutrinos and $\mu \rightarrow e, \gamma$, Nucl. Phys. B 618 (2001) 171 [hep-ph/0103065] [INSPIRE].

[90] M.E. Gómez, T. Hahn, S. Heinemeyer and M. Rehman, Higgs masses and Electroweak Precision Observables in the Lepton-Flavor-Violating MSSM, Phys. Rev. D 90 (2014) 074016 [arXiv: 1408.0663] [INSPIRE].

[91] J. Hisano, T. Moroi, K. Tobe and M. Yamaguchi, Lepton flavor violation via right-handed neutrino Yukawa couplings in supersymmetric standard model, Phys. Rev. D 53 (1996) 2442 [hep-ph/9510309] [INSPIRE].

[92] M.E. Gomez, S. Heinemeyer and M. Rehman, Effects of Sfermion Mixing induced by RGE Running in the Minimal Flavor Violating CMSSM, Eur. Phys. J. C 75 (2015) 434 [arXiv: 1501.02258] [INSPIRE].

[93] W. Buchmüller, P. Di Bari and M. Plümacher, Leptogenesis for pedestrians, Annals Phys. 315 (2005) 305 [hep-ph/0401240] [INSPIRE].

[94] C.S. Fong, E. Nardi and A. Riotto, Leptogenesis in the Universe, Adv. High Energy Phys. 2012 (2012) 158303 [arXiv:1301.3062] [INSPIRE].

[95] P.F. Harrison, D.H. Perkins and W.G. Scott, Tri-bimaximal mixing and the neutrino oscillation data, Phys. Lett. B 530 (2002) 167 [hep-ph/0202074] [INSPIRE].

[96] G. Bertone, D.G. Cerdeno, M. Fornasa, R. Ruiz de Austri, C. Strege and R. Trotta, Global fits of the CMSSM including the first LHC and XENON100 data, JCAP 01 (2012) 015 [arXiv:1107.1715] [INSPIRE].

[97] C. Strege, G. Bertone, F. Feroz, M. Fornasa, R. Ruiz de Austri and R. Trotta, Global Fits of the CMSSM and NUHM including the LHC Higgs discovery and new XENON100 constraints, JCAP 04 (2013) 013 [arXiv:1212.2636] [INSPIRE].

[98] G. Bertone et al., Global analysis of the pMSSM in light of the Fermi GeV excess: prospects for the LHC Run-II and astroparticle experiments, JCAP 04 (2016) 037 [arXiv: 1507.07008] [INSPIRE].

[99] B.C. Allanach, SOFTSUSY: a program for calculating supersymmetric spectra, Comput. Phys. Commun. 143 (2002) 305 [hep-ph/0104145] [InSPIRE].

[100] http://projects.hepforge.org/softsusy/.

[101] G. Bélanger, F. Boudjema, A. Pukhov and A. Semenov, MicrOMEGAs 2.0: A Program to calculate the relic density of dark matter in a generic model, Comput. Phys. Commun. $\mathbf{1 7 6}$ (2007) 367 [hep-ph/0607059] [INSPIRE].

[102] http://lapth.in2p3.fr/micromegas/. 
[103] P. Gondolo, J. Edsjo, P. Ullio, L. Bergstrom, M. Schelke and E.A. Baltz, DarkSUSY: Computing supersymmetric dark matter properties numerically, JCAP 07 (2004) 008 [astro-ph/0406204] [INSPIRE].

[104] http://www.darksusy.org/.

[105] F. Mahmoudi, SuperIso: A Program for calculating the isospin asymmetry of $B \rightarrow K^{*} \gamma$ in the MSSM, Comput. Phys. Commun. 178 (2008) 745 [arXiv:0710.2067] [INSPIRE].

[106] F. Mahmoudi, SuperIso v2.3: A Program for calculating flavor physics observables in Supersymmetry, Comput. Phys. Commun. 180 (2009) 1579 [arXiv:0808.3144] [INSPIRE].

[107] F. Feroz and M.P. Hobson, Multimodal nested sampling: an efficient and robust alternative to MCMC methods for astronomical data analysis, Mon. Not. Roy. Astron. Soc. 384 (2008) 449 [arXiv: 0704.3704] [INSPIRE].

[108] F. Feroz, M.P. Hobson and M. Bridges, MultiNest: an efficient and robust Bayesian inference tool for cosmology and particle physics, Mon. Not. Roy. Astron. Soc. 398 (2009) 1601 [arXiv: 0809.3437] [INSPIRE].

[109] http://www.ft.uam.es/.

[110] HFLAV collaboration, Averages of b-hadron, c-hadron, and $\tau$-lepton properties as of 2018, arXiv: 1909.12524 [INSPIRE].

[111] ATLAS collaboration, Interpretations of SUSY Searches in ATLAS with Simplified Models, in Meeting of the APS Division of Particles and Fields, 10, 2011 [arXiv:1110.0282] [INSPIRE].

[112] CMS collaboration, Interpretation of Searches for Supersymmetry with Simplified Models, Phys. Rev. D 88 (2013) 052017 [arXiv:1301.2175] [InSPIRE].

[113] F. Ambrogi et al., SModelS v1.2: long-lived particles, combination of signal regions, and other novelties, Comput. Phys. Commun. 251 (2020) 106848 [arXiv:1811.10624] [INSPIRE].

[114] S. Kraml et al., SModelS: a tool for interpreting simplified-model results from the LHC and its application to supersymmetry, Eur. Phys. J. C 74 (2014) 2868 [arXiv:1312.4175] [INSPIRE].

[115] F. Ambrogi, S. Kraml, S. Kulkarni, U. Laa, A. Lessa and W. Waltenberger, On the coverage of the pMSSM by simplified model results, Eur. Phys. J. C $\mathbf{7 8}$ (2018) 215 [arXiv: 1707.09036] [INSPIRE].

[116] A. Djouadi, M.M. Muhlleitner and M. Spira, Decays of supersymmetric particles: The Program SUSY-HIT (SUspect-SdecaY-HDECAY-InTerface), Acta Phys. Polon. B 38 (2007) 635 [hep-ph/0609292] [INSPIRE].

[117] T. Sjöstrand et al., An introduction to PYTHIA 8.2, Comput. Phys. Commun. 191 (2015) 159 [arXiv: 1410.3012] [INSPIRE].

[118] CMS collaboration, Search for supersymmetry in multijet events with missing transverse momentum in proton-proton collisions at 13 TeV, Phys. Rev. D 96 (2017) 032003 [arXiv: 1704.07781] [INSPIRE].

[119] CMS collaboration, Search for new phenomena with the $M_{\mathrm{T} 2}$ variable in the all-hadronic final state produced in proton-proton collisions at $\sqrt{s}=13$ TeV, Eur. Phys. J. C 77 (2017) 710 [arXiv: 1705.04650] [INSPIRE]. 
[120] ATLAS collaboration, Search for squarks and gluinos with the ATLAS detector in final states with jets and missing transverse momentum using $\sqrt{s}=8 \mathrm{TeV}$ proton-proton collision data, JHEP 09 (2014) 176 [arXiv:1405.7875] [INSPIRE].

[121] CMS collaboration, Search for direct production of supersymmetric partners of the top quark in the all-jets final state in proton-proton collisions at $\sqrt{s}=13 \mathrm{TeV}$, JHEP 10 (2017) 005 [arXiv: 1707.03316] [INSPIRE].

[122] CMS collaboration, Search for supersymmetry in proton-proton collisions at $13 \mathrm{TeV}$ using identified top quarks, Phys. Rev. D 97 (2018) 012007 [arXiv:1710.11188] [InSPIRE].

[123] CMS collaboration, Search for top squark pair production in pp collisions at $\sqrt{s}=13 \mathrm{TeV}$ using single lepton events, JHEP 10 (2017) 019 [arXiv:1706.04402] [INSPIRE].

[124] CMS collaboration, Combined search for electroweak production of charginos and neutralinos in proton-proton collisions at $\sqrt{s}=13$ TeV, JHEP 03 (2018) 160 [arXiv: 1801.03957] [INSPIRE].

[125] LUX-ZEPLIN collaboration, Projected WIMP sensitivity of the LUX-ZEPLIN dark matter experiment, Phys. Rev. D 101 (2020) 052002 [arXiv:1802.06039] [InSPIRE].

[126] DARWIN collaboration, DARWIN: towards the ultimate dark matter detector, JCAP 11 (2016) 017 [arXiv: 1606.07001] [INSPIRE]. 